This item was submitted to Loughborough's Research Repository by the author.

Items in Figshare are protected by copyright, with all rights reserved, unless otherwise indicated.

\title{
CONVEX (CONtinuously Varied EXtrusion): a new scale of design for additive manufacturing
}

PLEASE CITE THE PUBLISHED VERSION

https://doi.org/10.1016/j.addma.2020.101576

PUBLISHER

Elsevier

VERSION

AM (Accepted Manuscript)

PUBLISHER STATEMENT

This paper was accepted for publication in the journal Additive Manufacturing and the definitive published version is available at https://doi.org/10.1016/j.addma.2020.101576.

LICENCE

CC BY-NC-ND 4.0

\section{REPOSITORY RECORD}

Moetazedian, Amirpasha, Anthony Setiadi Budisuharto, Vadim Silberschmidt, and Andy Gleadall. 2020. "CONVEX (continuously Varied Extrusion): A New Scale of Design for Additive Manufacturing". Loughborough University. https://hdl.handle.net/2134/13286426.v1. 


\section{CONVEX (CONtinuously Varied EXtrusion): a new scale of design for additive manufacturing}

Amirpasha Moetazedian ${ }^{\mathrm{a}}$, Anthony Setiadi Budisuharto ${ }^{\mathrm{b}}$, Vadim V. Silberschmidt ${ }^{\mathrm{a}}$ and Andrew Gleadall ${ }^{*}$

a Wolfson School of Mechanical, Electrical and Manufacturing Engineering, Loughborough University, Loughborough, LE11 3TU, UK

b Department of Materials Science and Engineering, City University of Hong Kong, Hong Kong, 83 Tat Chee Avenue, Kowloon 999077, Hong Kong Special Administrative Region, China

*Corresponding author - Email: A.Gleadall@lboro.ac.uk; Tel: +441509 227578

\section{AUTHOR VERSION}

\section{Graphical abstract}

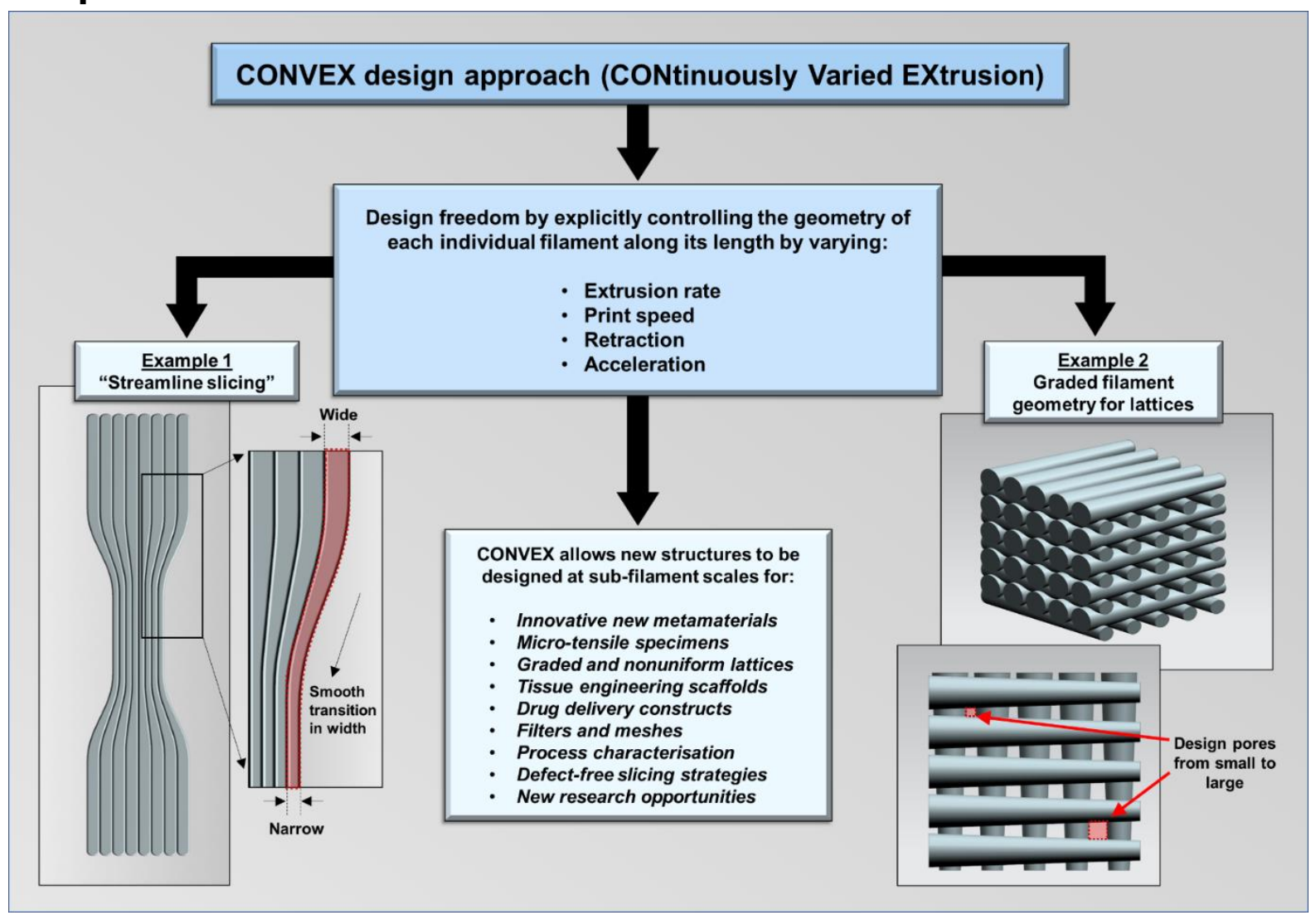

\section{Abstract}

This study introduces a new microscale design approach, CONVEX, based on the idea of CONtinuously Varying EXtrusion widths of deposited filaments in material extrusion additive manufacturing (MEAM). The CONVEX design approach breaks free from the traditional 3D printing workflow of filling a CAD-model volume with stacked extruded filaments of constant width and height. Instead, the geometry of each filament is explicitly designed over its entire 
length. The concept may disrupt a wide range of applications in both the short and long term. In the least ambitious short-term implementation of CONVEX, its principles can be integrated into 3D printing slicing software to allow a geometric form to be fitted by streamlined filaments, with constantly varying widths as required to match the overall geometry (referred to as "streamlined slicing"). The use of continuous streamlined filaments, as opposed to frequently changing the number of filaments, improved the quality of manufactured parts by eradicating voids and defects, which are known to cause critical stress concentrations in specimens for tensile testing. At the other end of the scale, in the most disruptive implementation of the CONVEX design approach, entire new material structures and product types will be enabled, with feature size-scales perhaps an order of magnitude lower than that permitted by present design rules. This will enable new innovative metamaterials and is particularly appropriate for high-value applications such as advanced filtering, tissue engineering, drug delivery, microfluidics or electronics, where the geometries of individual extruded filaments (or deliberate inter-filament pores) form the functional design geometry. To prove the technical feasibility of CONVEX, this study includes rigorous experimental work to characterise how instantaneous changes to MEAM process parameters (e.g. speed, acceleration, extrusion rate and retraction) enable highly controllable and dynamic variation of the width of extruded filaments (from $75 \%$ to $250 \%$ of nozzle diameter). The concept is proven for multiple materials, layer heights, extrusion temperatures, nozzle sizes, and for both Bowden and direct-drive printer types. The Bowden printer was found to be an order of magnitude less responsive to changes in extrusion rate than the direct-drive printer. Case studies demonstrate the CONVEX design approach, which has already enabled breakthrough micro-mechanical research for MEAM. Industrial implications are discussed along with the potential for translation to other manufacturing processes.

Keywords: additive manufacturing; metamaterials; microscale design; Bowden tube; subfilament design.

\section{Nomenclature:}

ABS - Acrylonitrile butadiene styrene

AM - Additive manufacturing

CAD - Computer-aided-design

CONVEX - CONtinuously Varied EXtrusion design approach

EFW - Extruded-filament width

FFF - Filament fused fabrication

FDM - Fused deposition modelling

MEAM - Material extrusion additive manufacturing

PEEK - Polyetheretherketone

PLA - Polylactide

PCL - Polycaprolactone

$\mathrm{ROI}$ - Region of interest

$\Delta \mathrm{W}_{\mathrm{s}}$ - Disruption of EFW (magnitude change) in response to instantaneous changes in a printing parameter. 
$\Delta \mathrm{W}_{\mathrm{r}}$ - Recovery of EFW after an imbalance is introduced - typically caused by a mismatch between the current extrusion pressure and the extrusion pressure required to achieve the desired extrusion rate.

$D_{s}$ - Normalised distance for a magnitude change in EFW in response to instantaneous change in printing parameter.

$D_{r}$ - Normalised distance for recovery of EFW.

\section{Introduction}

Additive manufacturing (AM) technologies have shown rapid and consistent development due to their ability to facilitate fast product development and produce structures and metamaterials that are not possible with conventional subtractive manufacturing processes $[1,2]$. Consequently, the popularity of $\mathrm{AM}$ has grown in a range of sectors [3-5]. In particular, the affordability and material-versatility of material extrusion additive manufacturing (MEAM) - also known as fused filament fabrication (FFF) or fused deposition modelling (FDM) resulted in its use for a variety of applications such as biomedical, automotive and aerospace [2,5-7]. Many thermoplastic polymers are currently being used in MEAM, including acrylonitrile butadiene styrene (ABS), polylactide (PLA), polyamide (nylon), polycaprolactone (PCL) and polyether-ether-ketone (PEEK) [8-12]. Importantly, this technology allows the production of complex and intricate parts, metamaterials and structures that are not possible with injection moulding or other conventional manufacturing processes [5,13].

MEAM of thermoplastic polymer is achieved by depositing material onto the print platform layer-by-layer. The process starts with feeding the filament into a melt chamber, set above the melting point of the polymer, via a feeding mechanism consisting of rollers and gears (Figure 1). The molten polymer is then extruded through a nozzle onto the print platform [3]. The nozzle's print path defines the physical embodiment of the designed part or structure; typically, one layer of a product is produced by moving the nozzle in the $x / y$ directions (Figure 1) before incremental movement of the print platform in the $z$ direction. The typical workflow for production of a 3D-printed part is: (i) generating a CAD model; (ii) exporting an STL file; (iii) generating the print path in slicer software; (iv) exporting machine control commands (G-Code) and (v) layer-by-layer manufacture.

MEAM 3D printers can be categorised into two main types based on their feeding mechanism: (a) direct drive and (b) Bowden tube (Figure 1) [14]. In both systems, the feeding mechanism pushes the filament into the melt chamber and generates the pressure required to extrude material through the nozzle. For direct-drive systems (Figure 1a), the feeder is attached directly above the printhead. For Bowden printers (Figure 1b), it is placed on the printer frame and the filament is pushed through a Bowden tube. In this setup, the distance from the feeder to the nozzle is greatly increased (potentially ten times longer) by comparison to direct-drive printers [14]. Therefore, Bowden printers can suffer from unresponsiveness: for instance, when the extrusion rate changes in the G-Code, there may be a lag before a change to the physical extrusion rate from the nozzle. 


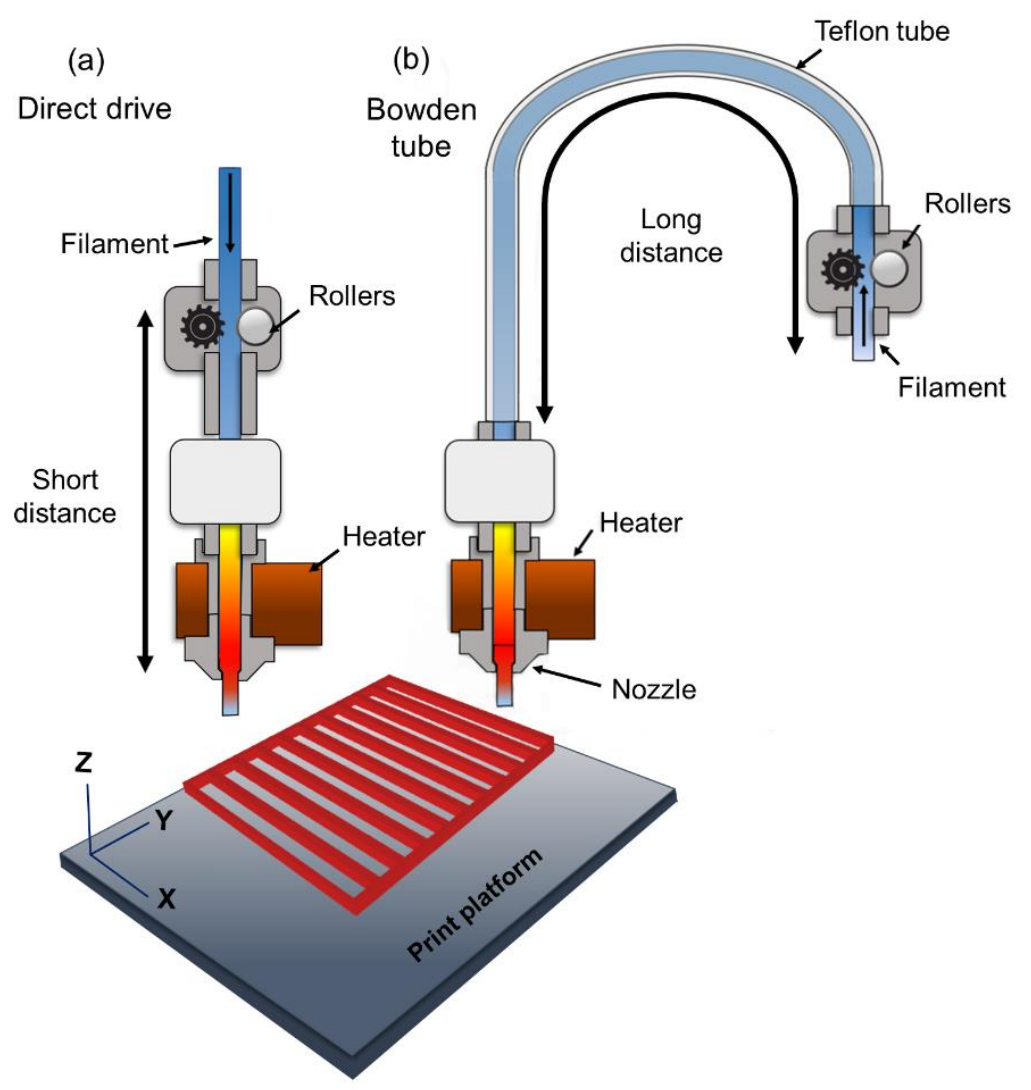

Figure 1 Schematic indicating differences between direct-drive (a) and Bowden (b) 3D printers.

Most studies in the literature [15-17] focused on the effect of slicer-software printing parameters on the mechanical performance or surface quality of 3D-printed parts. In contrast, there are limited studies investigating the effect of different parameters on the geometry of single extruded filaments $[9,18]$. This is partially due to the traditional 3D printing workflow of using computer-aided-design (CAD) to design the part and then employing slicing software to adjust the printing parameters [19]. This approach limits the potential to have a full control over the printing process and print path. A few studies $[9,18,20]$ used inhouse software to directly control the $3 \mathrm{D}$ printing process and clearly demonstrated the benefit of this approach. For example, Geng et al. [9] found the print speed to significantly affect the microstructure and dimensions of extruded PEEK filaments. Barrios and Romero [21] showed that acceleration influenced the surface roughness of 3D-printed parts. However, current studies investigating the geometry of extruded filaments considered steady-state printing conditions. This study investigates for the first time transitions in microscale filament geometry in response to a range of important control parameters (i.e. the transition between two steady-state conditions when printing a single filament), including: print speed, extrusion rate, retraction/un-retraction, acceleration and jerk. Furthermore, important influences of the feeding-system design (direct-drive versus Bowden) are analysed.

The need for this new investigation spans a range of fields from high-performance applications to new lattice-like structures or metamaterials, where geometry at the scale of single filaments or smaller, without defects, is important [22,23]. Existing print path strategies do not allow the full potential of 3D printers to be realised since they consider each extruded 
filament to have a constant width, and the part is effectively filled by positioning filaments side by side (or according to the chosen infill pattern).

Here, new understanding is developed for dynamic filament geometry (first three results sections) to support a new design approach called CONVEX (CONtinuously Varied EXtrusion), in which the widths of individual extruded filaments are designed to vary over their length (final results section). Case studies show how the CONVEX approach enables (i) new printpath-generation algorithms (for larger parts) or (ii) an entirely new scale of structural design (for intricate structures), which has ground-breaking potential for a wide range of fields, as already demonstrated by the CONVEX approach enabling technical breakthroughs in process and material understanding [24]. This study demonstrates the potential for a step-change in design capabilities and paves the way for a new generation of 3D printed metamaterials and structures. The versatility of the suggested concept is demonstrated by investigating different thermoplastic polymers (PLA, ABS and nylon), extrusion temperatures, layer heights and feeder mechanisms. The potential of the CONVEX design approach for industrial applications and other manufacturing processes is discussed.

\section{Materials and methods}

This section presents the CONVEX design approach and the method used to characterise dynamic process capabilities.

\subsection{CONVEX design approach}

The CONVEX design approach is based on designing at the sub-filament scale by varying the geometry of individual filaments along their length, as opposed to the conventional approach utilising filaments with a constant cross-section.

By arranging extruded filaments with a constant cross section to fill a CAD-model volume (a dogbone specimen for tensile testing is used for demonstration purposes), the discrete change in the number of filaments limits the design resolution and introduces defects/pores (Figure 2a), which were shown to act as critical stress concentrators for a range of print-path designs $[25,26]$. Adding so-called "perimeters" to the print path does not solve the problem; it simply shifts the defects from the surface to internal regions. Using the CONVEX design approach, streamlined filaments can be designed with a deliberate continuously varying width to fit the dogbone geometry, as shown in Figure $2 \mathrm{~b}$, eliminating voids/defects.

The change in width can be achieved by controlling speed and/or extrusion rate to allow the polymer material to spread to the desired width. The height of the filament is kept constant because it is governed by the space between the top of the previous layer's filaments and the bottom of the nozzle. Aside from improving the manufacturing quality, the CONVEX design approach also enables completely new design opportunities below the length-scale of individual filaments (e.g. to develop new structures and metamaterials). As an example, conventional design of tissue engineering scaffolds or similar structures uses log-pile stacking of filaments with unvarying geometry (Figure 2c). Therefore, pores between the extruded filaments are of a constant size. 

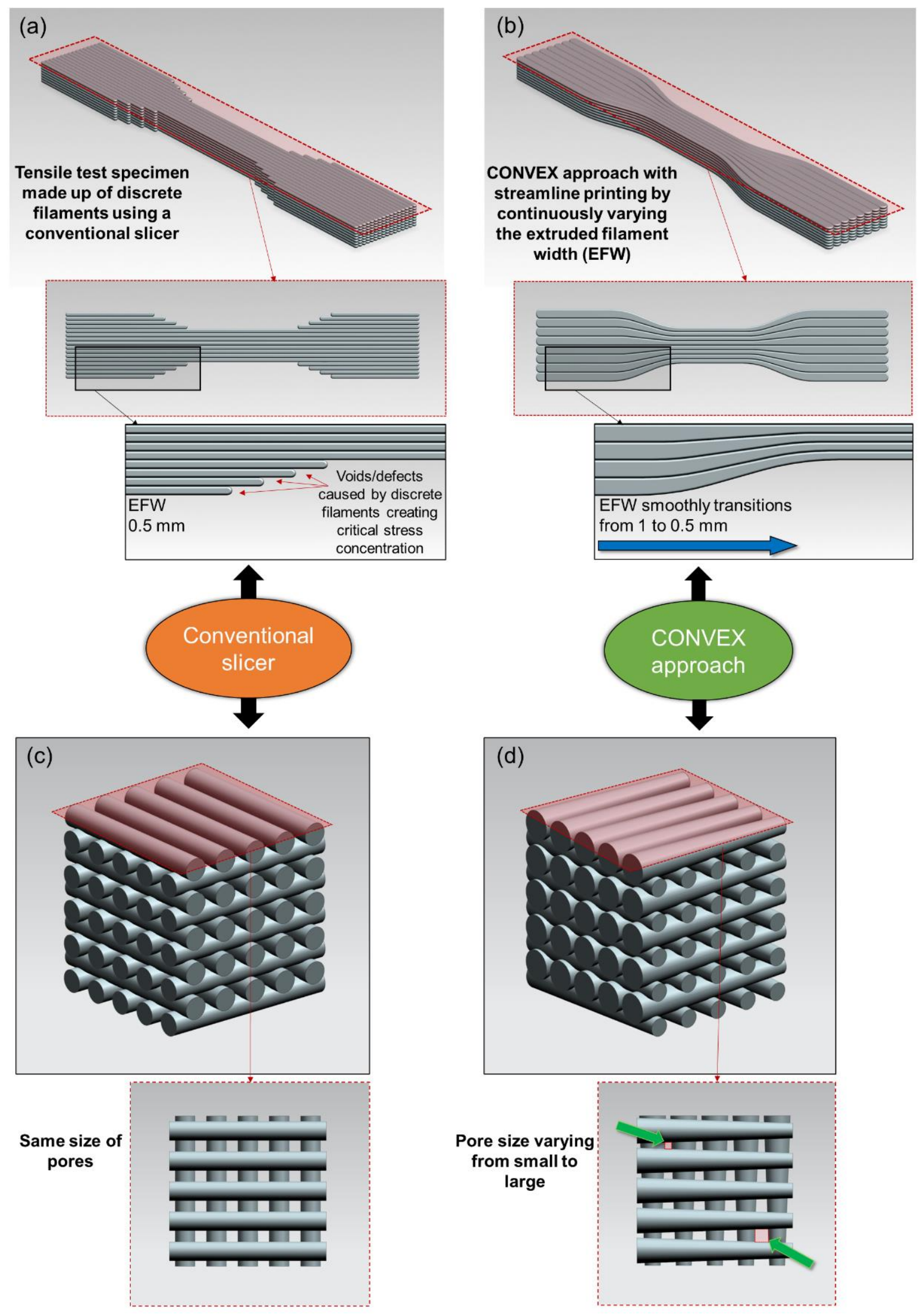

Figure 2 CAD models exemplifying CONVEX design approach. Designs for tensile test specimens and scaffold structures are shown for conventional (a and c) and CONVEX (b and d) design approaches, with geometry of individual filaments (extrusion width) deliberately designed to vary over each filament's length. 
Using the CONVEX design approach, each filament has a designed continuously varying width to achieve full control over spatial distribution of pores with different dimensions. A simple grading is shown in the schematic in Figure $2 \mathrm{~d}$; obviously, pore size could be adjusted in any manner, based on a greyscale image for example, which would not be possible by simply changing filament spacing in a conventional design. The CONVEX approach has already been used to design a new micro-tensile-testing specimen with varying extruded filament width (EFW), which allowed development of new fundamental understanding of micromechanics in AM [24,27].

The next sections describe the parametric experimental investigation of how printing parameters can be adjusted to achieve the CONVEX approach from a technical viewpoint.

\subsection{Parametric test-specimen design}

A custom design (Figure 3) was developed to analyse the effect of systematically varying a single parameter. In-house software ("FullControl GCODE Designer" - available from the corresponding author) was used to generate G-Code files for printing a 10-layer stack of single filaments. The print path is shown in Figure 3a (indicated by arrows). To normalise extrusion pressure after each line of print, a stabiliser line was used. The process was repeated 10 times (offset in y direction) to achieve a series of specimens in the region of interest $(\mathrm{ROI}$ - dashed box in Figure $3 \mathrm{~b})$. Each extruded filament was produced with three sections: sections 1 and 3 (10 mm long at the beginning and end) and section 2 (40 mm long in the middle) as shown in Figure 3c. Section 2 was printed with different printing parameters (e.g. print speed) compared to sections 1 and 3 to characterise the dynamic response of EFW. Figure $3 \mathrm{c}$ exemplifies the variation of the filament width in reponse to printing parameters: in that example, section 2 was printed at a faster speed than sections 1 and 3 , resulting in less time for material deposition and, therefore, a narrower EFW.

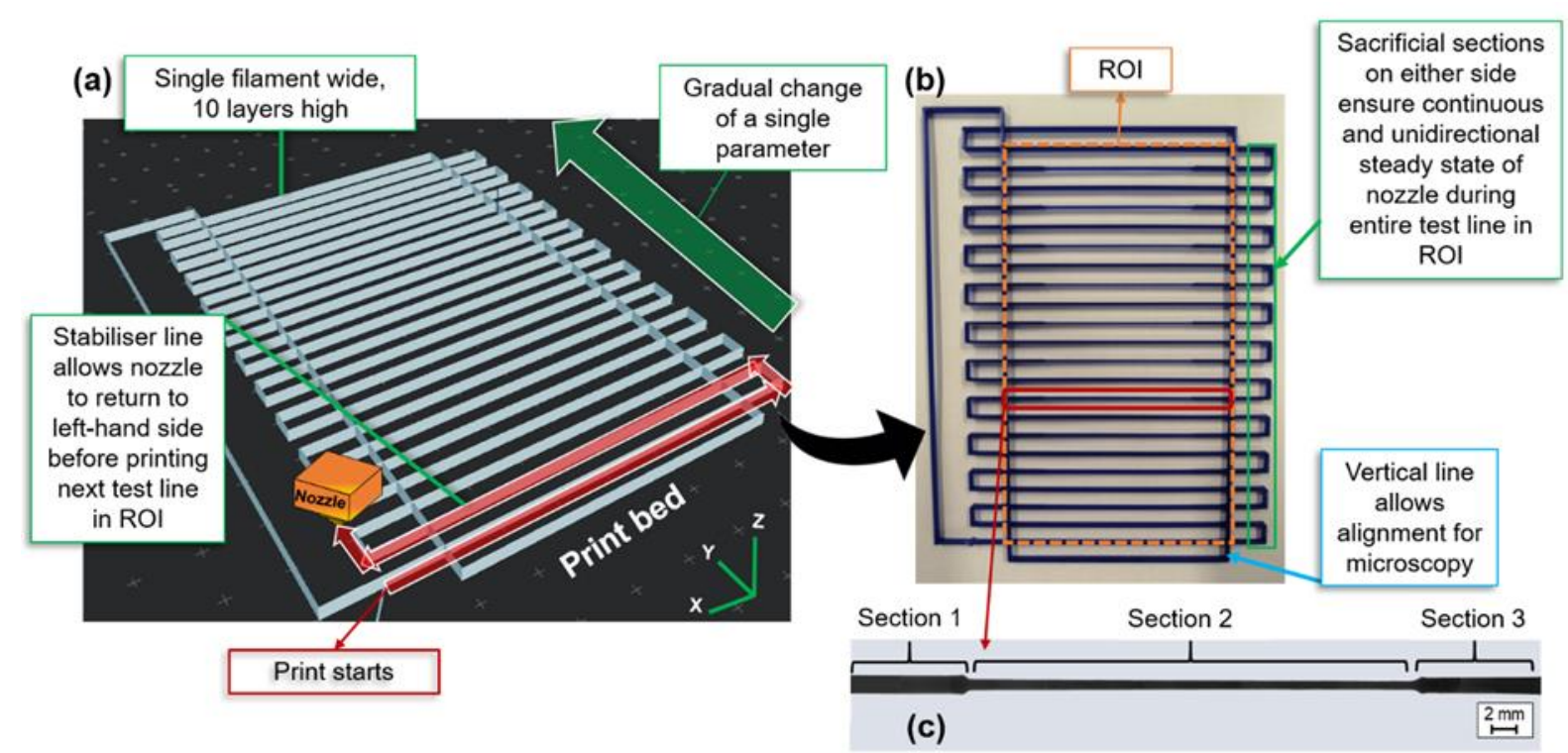

Figure 3 (a) Schematic of the design showing the nozzle's print path (red arrows) to investigate the effect of gradually changing a single printing parameter. (b) Image of the final 3D-printed part highlighting the region of interest (ROI) used to characterise filament geometry. (c) Optical micrograph showing the change in extruded filament width across the three sections. 


\subsection{Materials and 3D printing setup}

The testing design defined in Section 2.2 was printed using a Raise3D Pro2 (Raise3D ${ }^{\circledR}$, USA) system as a representative for direct-drive printers. The printer manufacturer's polylactide was used (Rasie3 $\mathrm{D}^{\circledR}$ Premium PLA); with printing parameters kept as default unless changed as shown in Table 1. The nozzle deposited a single filament, as defined in Section 2.2, using either a constant nominal width across all three sections (target EFW $=1$ ) or different widths (sections 1 and 3 target $E F W=1$; section 2 target $E F W=0.5$ ). The target EFW was physically implemented by adjusting the material's feed rate into the nozzle in GCode. Both design regimes were employed to characterise the effect of various printing parameters on the extruded filament's geometry, which are described in detail in Section 2.4. A $0.4 \mathrm{~mm}$ nozzle was used with extrusion temperature $205^{\circ} \mathrm{C}$ and layer height $0.2 \mathrm{~mm}$.

To investigate the difference between direct-drive and Bowden tube printers, an Ultimaker $2^{+}$ (Ultimaker ${ }^{\circledast}$, Netherlands) was used to represent latter systems. As with the former one, the printer manufacturer's polylactide was also used (Ultimaker ${ }^{\circledast} \mathrm{PLA}$ ) to print the same designs as those printed by the direct-drive system (defined in Section 2.2).

To investigate transferability of results to other materials, an alternative PLA supplier $\left(3 \mathrm{DXTECH}{ }^{\circledR}\right)$ and two alternative common polymers were used: ABS $\left(3 \mathrm{DXTECH}{ }^{\circledR}\right)$ and nylon (NOVAMID, PA6/66). A summary of parameters studied is given in Table 1. As highlighted to the left of the table, specific rows correlate to respective sections of the results and discussion. The first four rows cover the response of the direct-drive printer to changes in speed and retraction for constant and varied EFWs (results and discussion in Section 3.1). The next group of rows focus on the comparison between direct-drive and Bowden printers for a range of parameters (Section 3.2). In the third group, transferability of results to other materials and layer heights was studied (Section 3.3). The last row indicates that applicability of results was demonstrated in case studies (Section 3.4).

\subsection{Investigation of individual printing parameters}

Printing parameters investigated in this study are listed in Table 1, which also indicates the respective results section for each parameter. In each case, EFW was measured along the length of the filament and its variations were analysed with respect to instantaneous changes in print parameters (e.g. print speed).

\subsubsection{Print speed}

To investigate the effect of print speed (nozzle's movement in the direction of printing) on EFW, it was set to change from low to high and back to low in sections 1, 2 and 3, respectively, in (Figure 3c). The speed was always $1.0 \mathrm{~m}^{\mathrm{min}} \mathrm{m}^{-1}$ in sections 1 and 3 and ranged from 1.0 to $6.0 \mathrm{~m} \cdot \mathrm{min}^{-1}$ in section 2 (increments of $0.5 \mathrm{~m} \cdot \mathrm{min}^{-1}$ ). Acronyms are used to refer to each design in this study. The naming method is as follows: printing parameter (e.g. print speed) of section 1-section 2-section 3. For instance, to refer to samples with a print speed of $1.0 \mathrm{~m} \cdot \mathrm{min}^{-1}$ in sections $1 / 3$ and $4.0 \mathrm{~m} \cdot \mathrm{min}^{-1}$ in section 2 , the acronym 1.0-4.01.0 is used. 


\subsubsection{Acceleration and jerk}

Based on obtained results for print speed (Subsection 2.4.1), a reference speed of 3.0 $\mathrm{m} \cdot \mathrm{min}^{-1}$ for section 2 was employed with a range of accelerations to investigate the effect on the dynamic response of EFW. The default acceleration setting for many printers is between 2.0 and $5.0 \mathrm{m.s}^{-2}$ [14]; thus a broad range of acceleration was selected (0.05 to $6.0 \mathrm{m.s}^{-2}$ ).

The effect of "jerk", which refers to an instantaneous change in velocity, was investigated for $3.0 \mathrm{~m} \cdot \mathrm{min}^{-1}$ print speed in section 2 and $0.50 \mathrm{~m} . \mathrm{s}^{-2}$ acceleration. Typical jerk speeds are between 0.015 and $0.200 \mathrm{~m} . \mathrm{s}^{-1}$; so a wide range from 0.008 to $0.800 \mathrm{~m} . \mathrm{s}^{-1}$ was used.

\subsubsection{Retraction}

Another variable that significantly influences extrusion, is "retraction" (i.e. pulling material back into the nozzle). For the concepts discussed here, retraction (and un-retraction) may be useful to achieve sudden changes to EFW. Retraction was applied at the beginning of section 2 of specimens and was accompanied by un-retraction at the end of section 2 to ensure the same volume of material was extruded overall. A broad range of retraction values were used ( 0.02 to $0.20 \mathrm{~mm}$ for direct drive; 2 to $20 \mathrm{~mm}^{3}$ for Bowden). These ranges were chosen because they were either found in preliminary tests to be suitable for changing EFW by more than that required for the purposes of this study (direct drive), or because they considered a greater range than typically used (Bowden).

\subsubsection{Extrusion temperature and layer height}

To investigate the influence of extrusion temperature, experiments for speed and retraction (Sections 2.4.1 and 2.4.3) were repeated at lower and higher temperatures than the reference temperature $\left(195^{\circ} \mathrm{C}\right.$ and $215^{\circ} \mathrm{C}$ versus $205^{\circ} \mathrm{C}$, respectively) for both direct-drive and Bowden printers. This was done to investigate the sensitivity of findings and to demonstrate resilience and transferability of the CONVEX approach to different printing conditions. Further to this, specimens were printed with lower layer height $0.1 \mathrm{~mm}$ versus reference $0.2 \mathrm{~mm}$ ).

\subsection{Case studies}

Three case studies were used to demonstrate the CONVEX design approach versus a traditional 3D printing approach (CAD model > slicer software $>$ G-Code $>3 \mathrm{D}$ print). For all case studies, the Bowden printer was used. For the "traditional approach" in case studies, Cura software was used to slice CAD models and generate G-Code. For the CONVEX approach, in-house software ("FullControl GCODE Designer") was used to design the print path with explicit control over settings such as speed, extrusion rate and acceleration. Extrusion temperature was $205^{\circ} \mathrm{C}$ and layer height was $0.2 \mathrm{~mm}$ (first two case studies) or $0.3 \mathrm{~mm}$ (third case study). The printing quality of both methods were compared using a Zeiss Primotech optical microscope. Cura was used in this study because it is a powerful and well-respected slicer; the authors believe no alternative slicing software would be able to achieve better results for the structure sizes considered here. However, slicing software could be revised in the future to implement the CONVEX approach. A video of the third case study is supplied as supplementary information [28]. 
Table 1 List of parameters (along with corresponding results section numbers) used to assess changes in geometry of single extruded filaments for directdrive and Bowden printers (the bold italic font indicates the parameters that were the main focus for the respective row of the table).

\begin{tabular}{|c|c|c|c|c|c|c|c|c|c|c|c|c|}
\hline & \multirow{2}{*}{ Parameter } & \multicolumn{3}{|c|}{ Normalised target width } & \multicolumn{3}{|c|}{ Print speed $\left(\mathrm{m} \cdot \mathrm{min}^{-1}\right)$} & \multirow{2}{*}{$\begin{array}{c}\text { Retraction for } \\
\text { section } 2 \\
\text { (mm for direct-drive } \\
\& \mathrm{~mm}^{3} \text { for Bowden) }\end{array}$} & \multirow{2}{*}{ Material } & \multirow{2}{*}{$\begin{array}{c}\text { Temperature } \\
\left({ }^{\circ} \mathrm{C}\right)\end{array}$} & \multirow{2}{*}{$\begin{array}{c}\text { Acceleration } \\
\qquad\left(\mathrm{m} \cdot \mathrm{s}^{-2}\right)\end{array}$} & \multirow{2}{*}{$\begin{array}{l}\text { Layer } \\
\text { height } \\
(\mathrm{mm})\end{array}$} \\
\hline & & $\begin{array}{l}\text { Section } \\
1\end{array}$ & $\begin{array}{l}\text { Section } \\
2\end{array}$ & $\begin{array}{l}\text { Section } \\
3\end{array}$ & $\begin{array}{l}\text { Section } \\
1\end{array}$ & $\begin{array}{l}\text { Section } \\
2\end{array}$ & $\begin{array}{l}\text { Section } \\
3\end{array}$ & & & & & \\
\hline \multirow{5}{*}{ Section 3.1} & \multirow{2}{*}{ Speed } & 1 & 1 & 1 & 1 & $1-6$ & 1 & \multirow{2}{*}{0} & \multirow{7}{*}{ PLA } & \multirow{4}{*}{205} & \multirow{6}{*}{$\begin{array}{l}0.5 \text { (direct- } \\
\text { drive) \& } 3.0 \\
\text { (Bowden) }\end{array}$} & \multirow{8}{*}{0.2} \\
\hline & & 1 & 0.5 & 1 & 1 & $1-6$ & 1 & & & & & \\
\hline & \multirow{2}{*}{ Retraction } & 1 & 1 & 1 & 1 & 1 & 1 & $\begin{array}{l}0.02-0.2 \text { (direct- } \\
\text { drive) \& }\end{array}$ & & & & \\
\hline & & 1 & 0.5 & 1 & 1 & 1 & 1 & 2.0-20.0 (Bowden) & & & & \\
\hline & \multirow[b]{2}{*}{ Temperature } & 1 & 0.5 & 1 & 1 & $1-6$ & 1 & 0 & & \multirow[b]{2}{*}{$\begin{array}{l}195,205 \text { and } \\
215\end{array}$} & & \\
\hline \multirow[t]{2}{*}{ Section 3.2} & & 1 & 0.5 & 1 & 1 & 1 & 1 & $\begin{array}{l}0.02-0.2 \text { (direct-drive) } \\
\& 2.0-20.0 \text { (Bowden) }\end{array}$ & & & & \\
\hline & Acceleration & 1 & 0.5 & 1 & 1 & 3 & 1 & \multirow{3}{*}{0} & & 205 & $0.05-6.0$ & \\
\hline \multirow[t]{2}{*}{ Section 3.3} & Material & 1 & 0.5 & 1 & 1 & $1-6$ & 1 & & $\begin{array}{c}\text { PLA } \\
\text { ABS } \\
\text { Nylon }\end{array}$ & $\begin{array}{l}205 \\
240 \\
240\end{array}$ & \multirow[t]{2}{*}{$\begin{array}{c}0.5 \\
\text { (direct-drive) }\end{array}$} & \\
\hline & Layer height & 1 & 0.5 & 1 & 1 & $1-6$ & 1 & & PLA & 205 & & $0.1-0.2$ \\
\hline Section 3.4 & & & & & & & e & & & & & \\
\hline
\end{tabular}




\subsection{Image processing}

After printing, a SmartScope Flash 200 microscope (OGP, US) was used for dimensional measurements. An optical image was captured every $4.25 \mathrm{~mm}$ for each testing line to yield 14 images per specimen (Figure $4 a$ and b). A MATLAB script was developed to postprocess the data. All images were binarised and stitched together (Figure $4 \mathrm{c}$ and $\mathrm{d}$ ). Then, white pixels were counted to generate a plot of EFW versus distance. EFW was normalised with the initial width (beginning of Section 1). The distance was normalised with the nozzle diameter $(0.4 \mathrm{~mm})$.

\subsection{Quantitative analysis of dynamic change in EFW}

Two different modes of transition from wide to narrow EFW (and vice versa) between the three sections of specimen were identified:

- Transition mode I: associated with a sudden change in the normalised EFW, in response to instantaneous changes in a printing parameter (e.g. print speed). The change in width for this disruption is denoted as $\Delta \mathrm{W}_{\mathrm{s}}$ throughout this study (Figure 5).

- Transition mode II: associated with natural recovery of EFW to the target EFW. The change in width for recovery is denoted as $\Delta \mathrm{W}_{\mathrm{r}}$ (Figure 5). Natural recovery occurs if the target width is changed, as the extrusion process takes time to reach the new steady state. In addition, natural recovery occurs after a sudden disruption (transition mode I), when the extrusion process returns to the original width (before disruption).

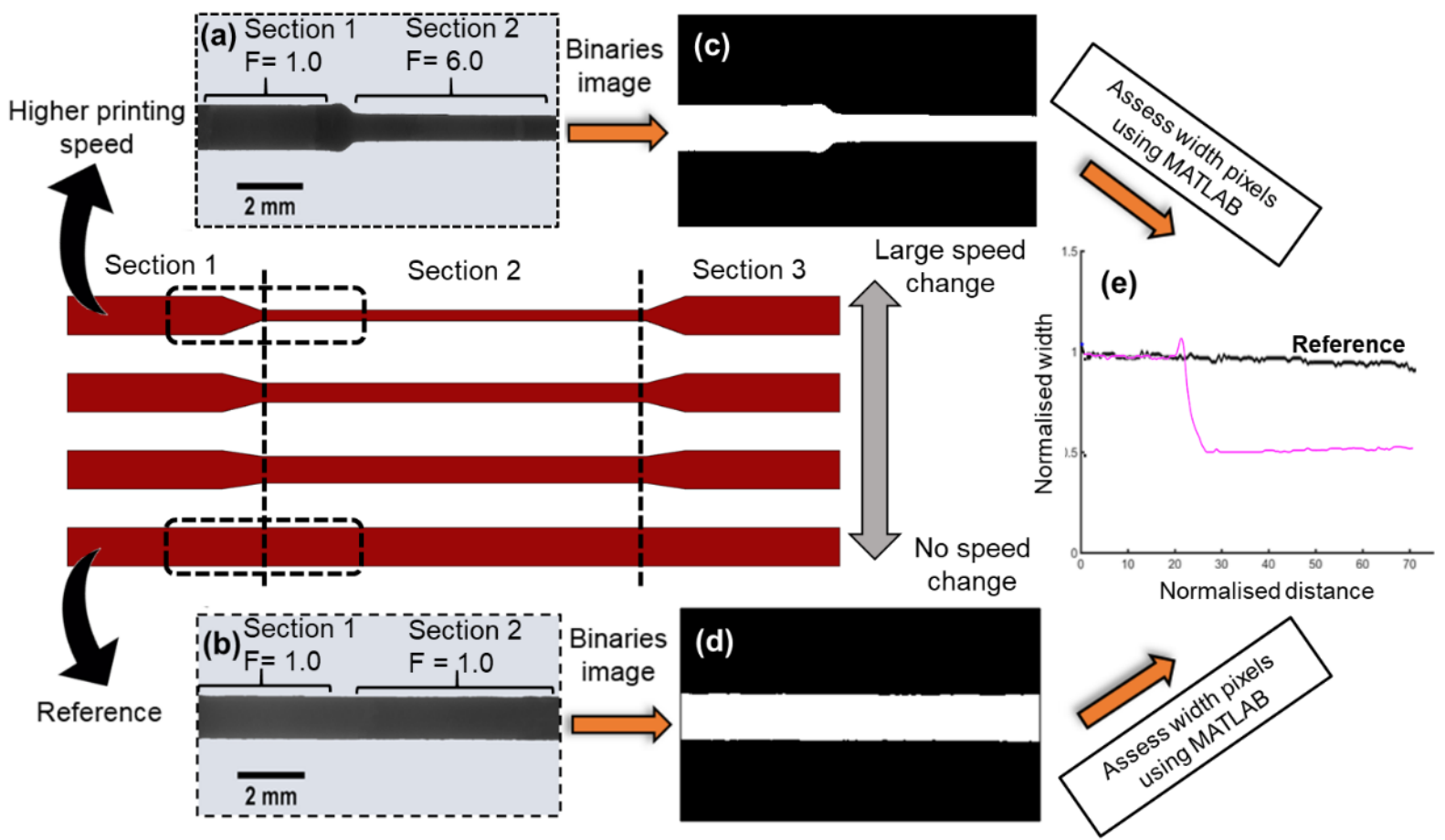

Figure 4 Schematic of workflow for measuring EFW. (a-b) Optical microscopy images were taken every $4.25 \mathrm{~mm}$ to yield 14 images per specimen. (c-d) A MATLAB script was used to binarise images and (e) the number of white pixels was counted to determine normalised EFW and its variation with normalised distance. 


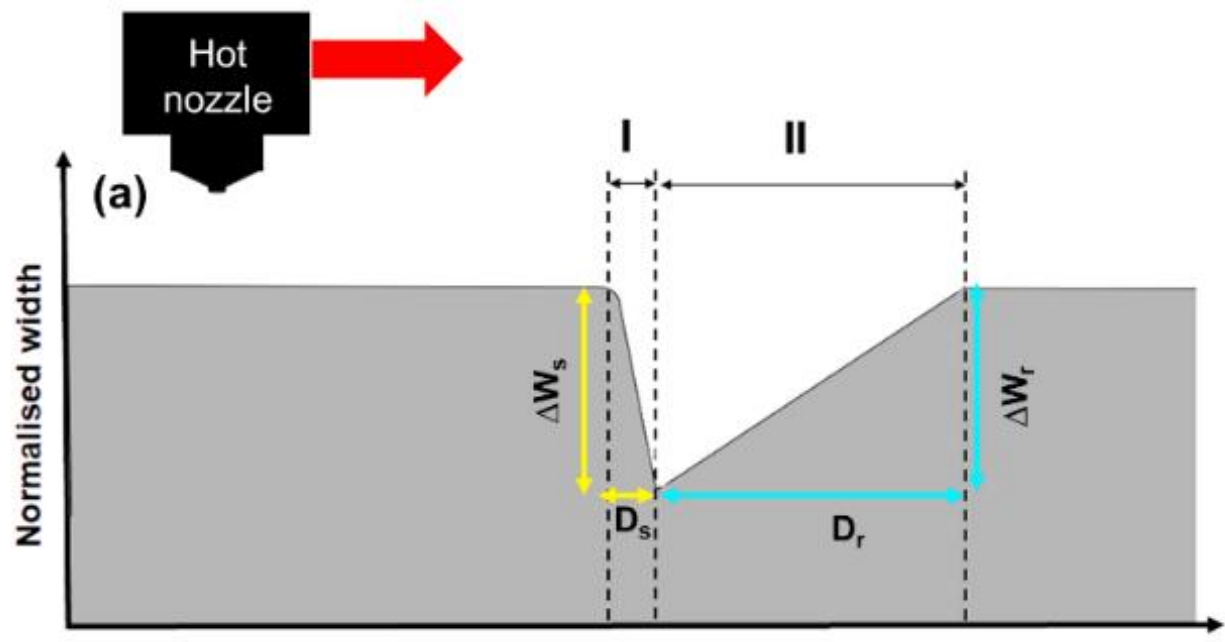

Normalised distance

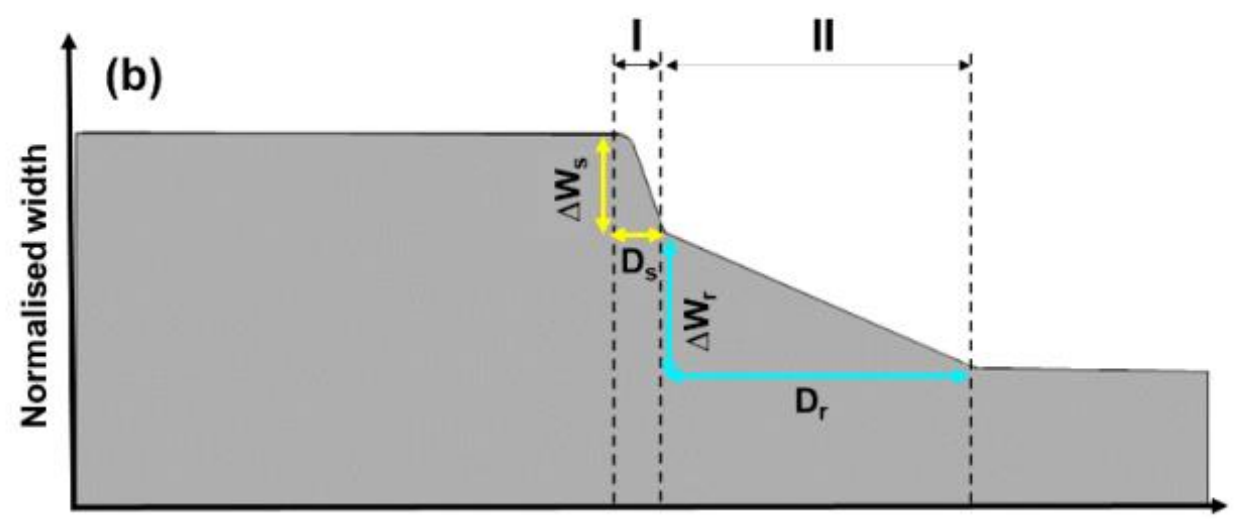

Normalised distance

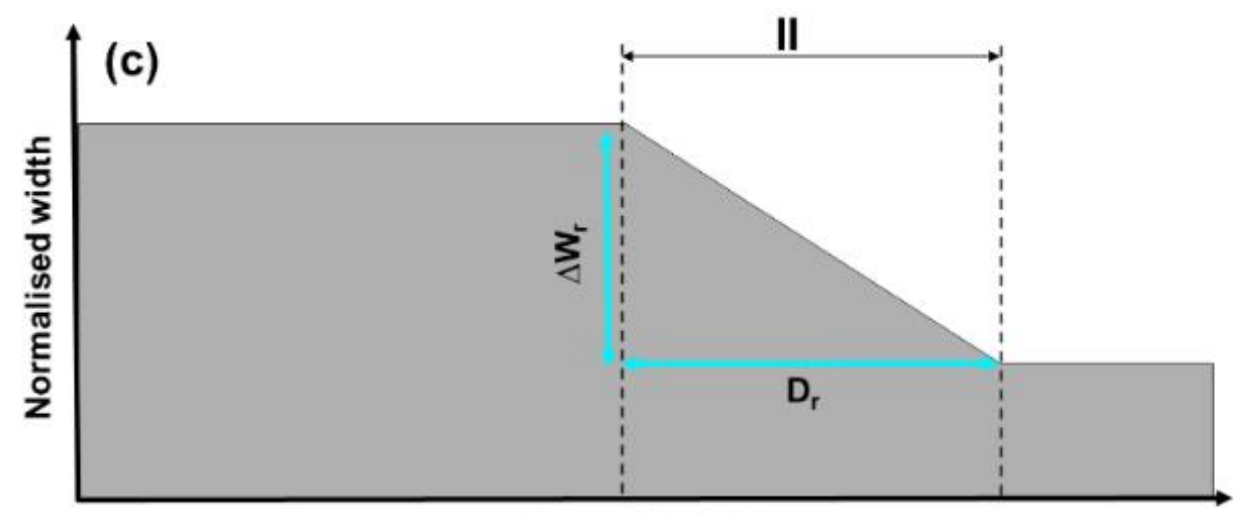

Normalised distance

Figure 5 Schematics for quantitative characterisation of changes to EFW. (a) For a constant target EFW an instantaneous change to a printing parameter results in a sudden change in EFW (transition mode I) followed by a gradual recovery back to the target EFW (transition mode II). (b) Similar to (a) but combined with a change to the target EFW. (c) Only changing the target EFW. 
The potential combinations of transition modes I and II are shown in Figure 5. When target width is kept constant, but the steady-state nature of the printing process is interrupted by an instantaneous change in a printing parameter, there is a sudden change in EFW followed by natural recovery (Figure 5a). When the target width of a filament is changed, but no other printing parameters are varied, steady-state conditions are interrupted and there is gradual recovery to the new target EFW (Figure 5c). When the target width is changed in combination with another printing parameter, there is a combination of a sudden change to

EFW and natural recovery to the new target EFW (Figure 5b). The gradient and magnitude of the sudden change and the natural recovery were calculated to allow quantitative analysis. To minimise the effect of minor experimental errors or fluctuations, the distance to achieve $95 \%$ of target EFW was considered for all quantifications.

\section{Results and Discussion}

The results and discussions are separated into four sections, as mapped out in Table 1:

- 3.1 - Dynamic response of EFW to changes in speed and retraction is investigated for the direct-drive printer.

- 3.2 - Responses are compared for the direct-drive and Bowden printers for a range of factors including speed, retraction, temperature and acceleration.

- 3.3 - Transferability and resilience of results are investigated in relation to material and layer height.

- $\quad 3.4$ - Case studies utilise the new understanding, developed in Sections 3.1-3.3, to demonstrate the CONVEX design approach to produce structures that are not possible with conventional 3D printing workflows/software. This section also discusses the suitability and applicability of results for a broad range of applications.

Before investigating the role of different parameters, first the effect of changing target EFW only (i.e. varying extrusion rate), while keeping all other parameters constant, was studied for both types of printer, as shown in Figure 6 . By changing the target width alone (to $50 \%$ of the initial width), the direct-drive printer achieved a gradual change in normalised width over a normalised distance of approximately 40 . The change was quite gradual because, when the target width was changed, the amount of filament being fed into the nozzle reduced from that point onwards, but over-extrusion occurred while residual pressure in the nozzle was gradually released. In contrast, the Bowden printer showed almost no response over a normalised distance of 125, which represents $50 \mathrm{~mm}$ of printing length for the $0.4 \mathrm{~mm}$ nozzle used in this study. This poor responsiveness of the Bowden printer was one of the justifications for investigating the effect of parameters other than target width. A technique called velocity painting [29] has been used to modify the surface texture of 3D-printed parts by changing the print speed at different locations to reduce EFW (at high print speed) or increase EFW (at low print speed). Therefore, in the next section (3.1.1), the effect of changing speed was examined. It is well known that retraction affects extrusion, so it was also studied (Section 3.1.2). 


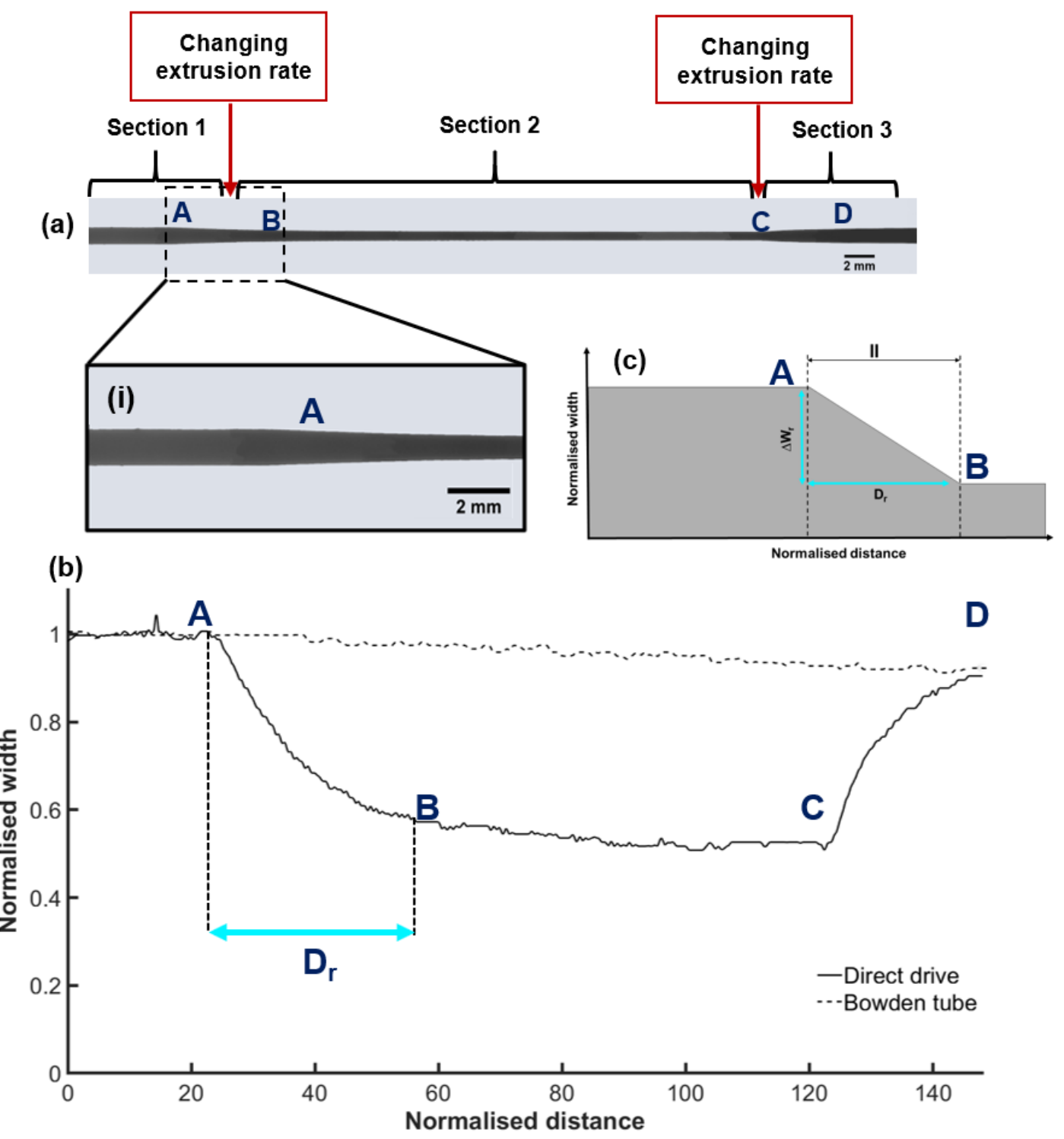

Figure 6 (a) Optical micrograph of entire specimen printed by direct-drive printer with zoomed-in image (i) for transition from section 1 to 2. (b) Comparison of almost negligible response of Bowden tube printer and slow response of direct-drive printer to change in target EFW from 1.0 to 0.5. (c) Schematic of transition mode, as defined in Figure 5.

\subsection{Effect of print speed and retraction (direct drive)}

\subsubsection{Print speed}

First, this section investigates the effect of the changing print speed in section 2 of the specimen while keeping the target EFW constant across all three sections (Figure 7b). The first specimen ( $I D=1.0-1.0-1.0$ ) showed no changes in the width, as expected, since the print speed and the target EFW were unchanged across all three sections. Increasing print speed from $1.0 \mathrm{~m} \cdot \mathrm{min}^{-1}$ to $6.0 \mathrm{~m} \cdot \mathrm{min}^{-1}$ in section 2 was associated with a sudden change $\left(\Delta \mathrm{W}_{\mathrm{s}}\right.$, transition mode I) in EFW visible in the optical micrographs (Figure 7i and iii). 
(a)
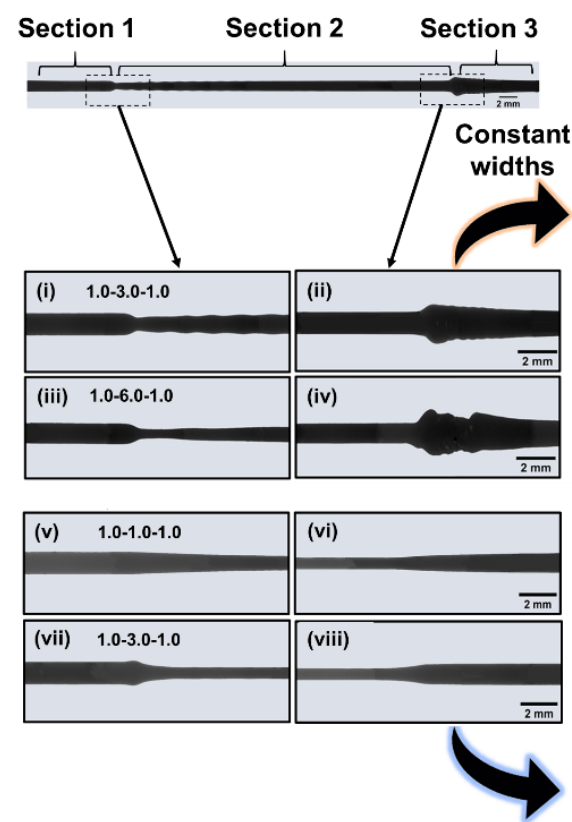

Different widths
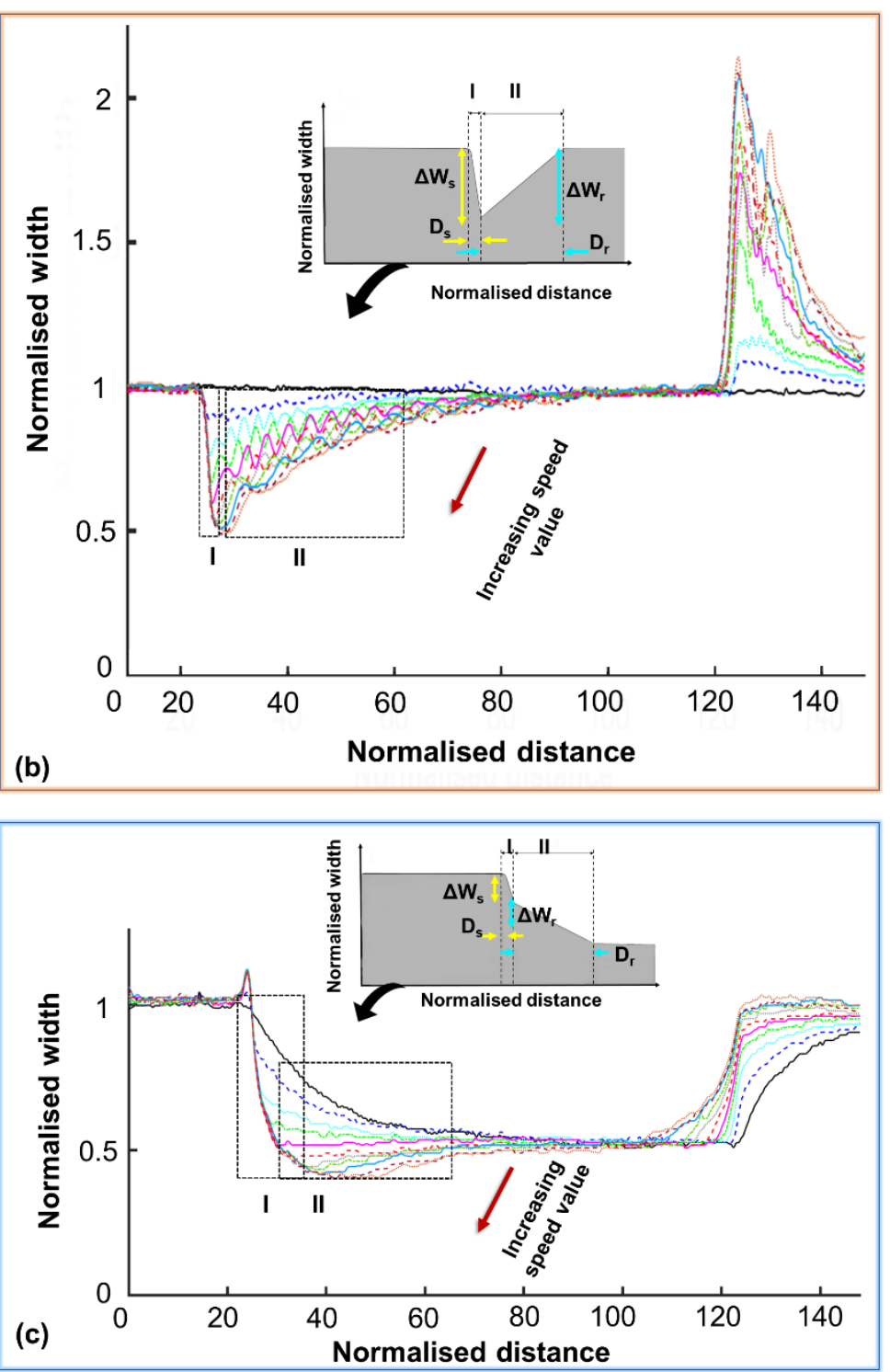

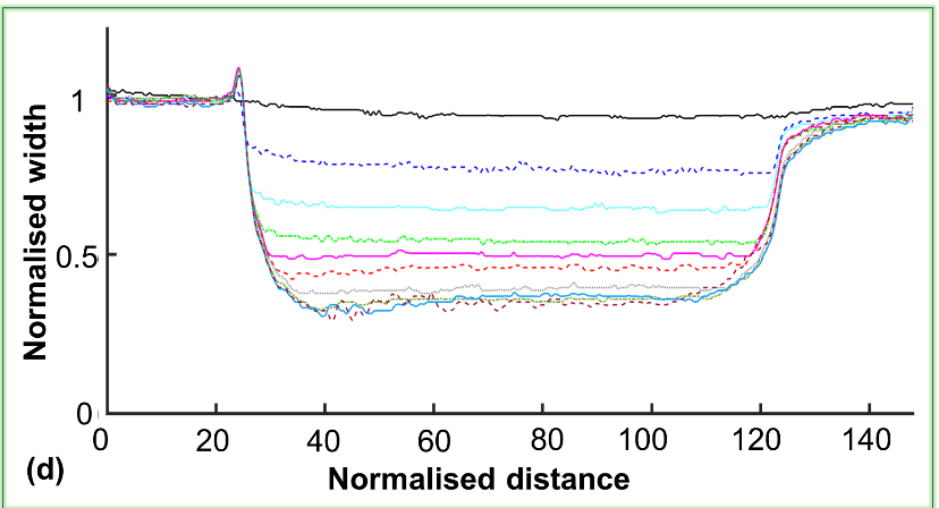

Speed ( $\left.\mathrm{m} \cdot \mathrm{min}^{-1}\right)$

-1.0-1.0-1.0

$\cdots-1.0-1.5-1.0$

$-1.0-2.0-1.0$

Adjusting widths in section 2 of

$-1.0-2.5-1.0$

specimen

$-1.0-3.0-1.0$

$--1.0-3.5-1.0$

$-1.0-4.0-1.0$

$-1.0-4.5-1.0$

$-1.0-5.0-1.0$

$--1.0-5.5-1.0$

$-1.0-6.0-1.0$

Figure 7 (a) Optical micrograph showing effect of changing print speed on EFW. (b) Evolution of normalised EFW with normalised distance along extruded-filament length for specimens with constant target EFW (i.e. 1.0) but print speed was varied in section 2 of specimens. (c) Similar to (b) but for target EFW $=0.5$ in section 2. (d) Matching speed and target width for rapid resumption of steadystate printing in section 2 based on results in (c). (i-iv) Micrographs for constant target EFW. (v-viii) Micrographs for varied target EFW. 
The obtained results contradict with findings by Geng et al. [9]. This could be explained since in that study [9], the print speed was slow by comparison to typical speeds in other studies, the fastest speed was eight times slower than the reference print speed in the current study. A comparison of the results with the specimen where only target width was changed (Figure 6b) clearly demonstrates the significant effect of print speed on dynamic changes to filament geometry. Increasing speed to $6.0 \mathrm{~m} \cdot \mathrm{min}^{-1}$ for section 2 of the specimen rapidly reduced EFW by approximately $50 \%$ over a transition distance of 2 (as opposed to 40 when the target EFW was changed alone in Figure 6). This rapid change (transition mode I) can be explained by the fact that the extrusion rate should increase linearly with speed to maintain a constant EFW; a sudden six-fold increase in the speed needed a respective increase in the amount of material fed into the nozzle. Therefore, at the beginning of section 2 of specimens, there was an imbalance between pressure inside the nozzle (that defined extrusion rate) and the printhead's acceleration. This resulted in the lagging of pressure inside the nozzle, causing under-extrusion (Figure 7b). As the pressure and acceleration resynchronised, the filament gradually recovered to the target EFW (transition mode II).

Another distinct feature observed was repetitive oscillations during the recovery, likely caused by vibration of the printhead. This is often attributed as the cause of printing defects called "ringing" [30]. Interestingly, oscillations of EFW had the greatest magnitude at midrange speeds (2.0-4.0 m.min $\left.{ }^{-1}\right)$ and were less evident at the highest speed $\left(6.0 \mathrm{~m} \cdot \mathrm{min}^{-1}\right)$, suggesting that certain speed ranges may cause resonant vibration of the printer.

After section 2, print speed was switched back to the reference value of $1.0 \mathrm{~m} \cdot \mathrm{min}^{-1}$ at the beginning of the section 3 . This was associated with a sudden increased in EFW (overextrusion), visible in Figure $7 \mathrm{~b}$ and optical micrographs (Figure 7ii and iv). The overextrusion resulted from a much higher pressure in the nozzle required at higher speeds (to achieve the related higher extrusion rates), which was gradually released by excessive material deposition after the nozzle suddenly changed to a slower speed. Higher print speeds in section 2 resulted in greater over-extrusions at the beginning of section 3 , as expected.

For the second design regime, the same print speed parameters were investigated, but the target EFW in the section 2 was halved compared to sections 1 and 3, to understand how combining the target width (Figure 6) and the print speed (Figure 7b) can enable control over the microscale geometry. The filament's geometry (Figure 7c) was completely different than those when changing the print speed only (Figure $7 \mathrm{~b}$ ) because the filament width approached the new target EFW in section 2. Similar to Figure $7 \mathrm{~b}$, the transition for changing the speed and width was separated into a sudden disruption $\left(\Delta \mathrm{W}_{\mathrm{s}}\right)$ and recovery $\left(\Delta \mathrm{W}_{\mathrm{r}}\right)$ towards the target EFW. In contrast to Figure $7 \mathrm{~b}$, the recovery portion (transition mode II) did not display repetitive oscillation patterns, with the print quality significantly improved as obvious from the optical micrographs (Figure 7v-viii). By changing print speed in section 2 , the new target EFW could be achieved relatively rapidly. At an optimal value of speed of approximately $3.0 \mathrm{~m} \cdot \mathrm{min}^{-1}$, the sudden change coincided with the new target width, therefore removing the need for recovery. In effect, the increase in speed (from section 1 to 2) demanded a higher extrusion pressure but the reduction in target EFW needed a lower extrusion pressure, with these two factors compensating each other. The second transition from section 2 to section 3 of the specimen - was also different compared to that for the constant-width design. No over-extrusion was observed for any specimen, while deceleration from a higher speed resulted in a more rapid initial transition, with a slower one for speeds in 
excess of $3.0 \mathrm{~m} \cdot \mathrm{min}^{-1}$. These observations highlight the complexity of the 3D printing process, which involves the interplay of the printhead motion, extrusion flowrate and the melt pressure.

By analysing the data (Figure 7c), the geometry of extruded filaments can be designed to have variable EFW along their length. This is demonstrated in Figure $7 d$, where the EFW in section 2 was set to be in the range between $30 \%$ and $100 \%$ of the width in sections 1 and 3 (representing a broad possible design window between $75 \%$ and $250 \%$ of nozzle diameter). To achieve this, the sudden change in EFW was identified in Figure 7c, and the target EFW of section 2 was adjusted to eliminate the need for a recovery transition. In other words, speeds and target widths were matched to allow rapid achievement of steady-state printing in section 2. The results presented in Figure $7 d$ indicated the feasibility of this hypothesis and potential for the CONVEX design approach to allow the design of geometries at the subfilament scale. This information could be useful for a range of industries, in which the microscale geometry of 3D printed parts is important, and for developers of printer hardware to quantitatively assess performance. It should be noted that a print speed investigation was also conducted for narrower target EFWs (the respective data are not shown) and exhibited similar trends, indicating potential transferability of the obtained results for a range of EFWs.

In summary, it was shown that print speed has an immediate and controllable short-term effect on filament geometry for the direct-drive printer (the Bowden printer is considered in Section 3.2). Combined with a change in target EFW (and thus a change in the continual extrusion rate), it is possible to change filament width with a high level of control.

\subsubsection{Retraction}

The second variable investigated for the direct-drive printer was retraction, which is widely used to prevent oozing of material that otherwise results in stringing [31,32]. Similar to print speed, the effect of retraction was considered for two sets of specimen designs with different target EFWs (constant and variable). For specimens that had the same target EFW in all three sections, results for retraction are shown in Figure $8 \mathrm{~b}$ and i-iv. As the retraction value increased from 0 to $0.20 \mathrm{~mm}$, EFW at section 2 reduced by up to $50 \%$ for a short period (Figure $8 \mathrm{i}$ and iii). The results showed a similar trend to changing the speed only (see Figure $7 \mathrm{~b})$ in terms of sudden change $\left(\Delta \mathrm{W}_{\mathrm{s}}\right)$ in the normalised EFW, although the reduction gradient was steeper for retraction than for changing speed. This could be explained by the difference in the mechanism of reducing filament width. Apparently, the acceleration of the printhead to faster speeds was less sudden than the retraction of filament, which is logical due to the high mass of the printhead for direct drive systems (since the extruder is mounted on the printhead). 
(a)
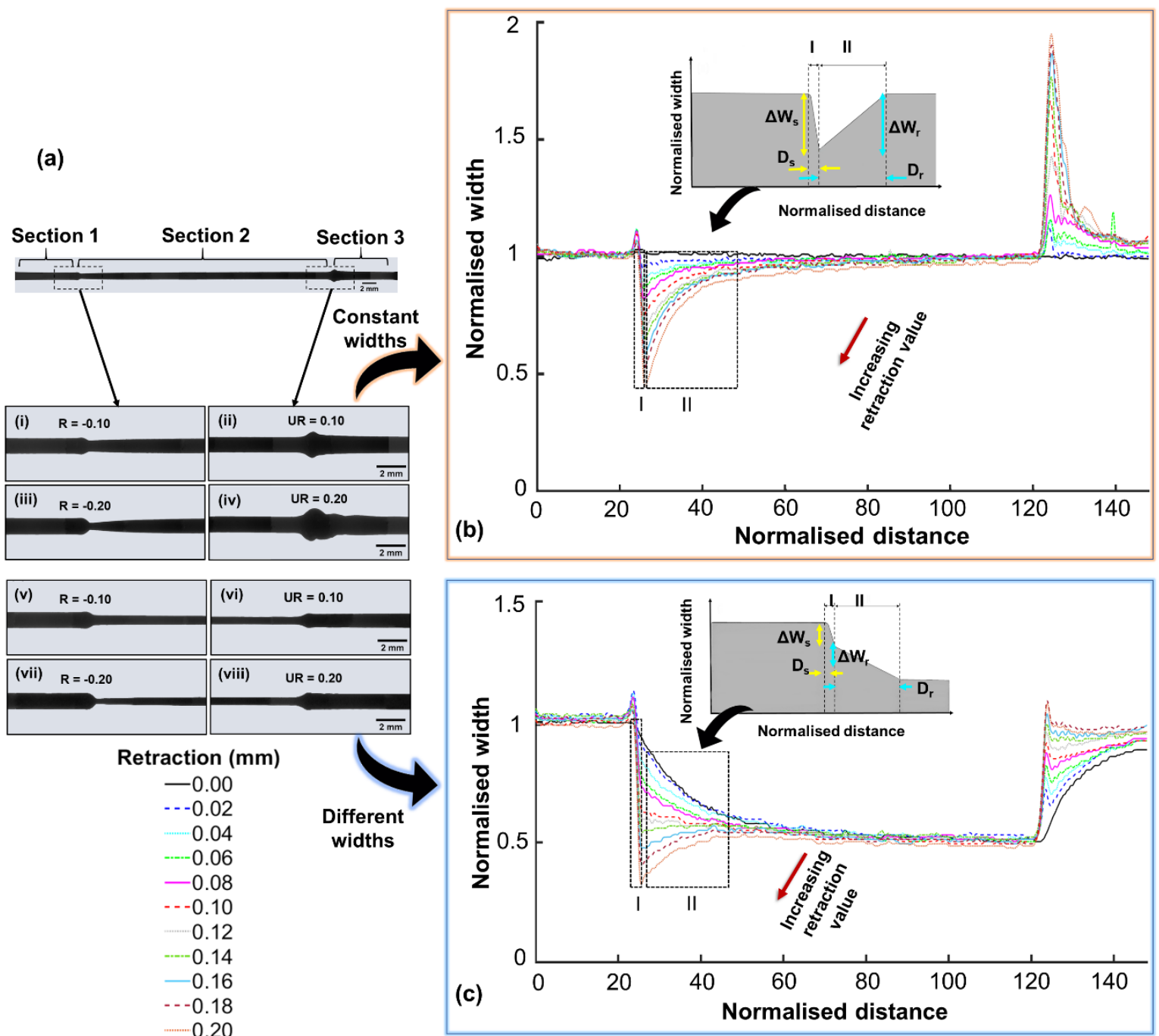

Figure 8 (a) Optical micrograph showing effect of retraction (left) and un-retraction (right) on EFW. (b) Evolution of normalised EFW with normalised distance along extruded-filament length for specimens with constant target EFW (1.0) and retraction/un-retraction applied at start/end of section 2, respectively. (c) Similar to (b) but target $E F W=0.5$ in section 2. ( $R=$ retraction; $U R=$ un-retraction). ( $i$ iv) Micrographs for constant target EFW. (v-viii) Micrographs for varied target EFW.

During the retraction process, as the length of retraction increased, a larger volume of material was pulled back into the nozzle and, thus, greater under-extrusion was observed (Figure 8). After retraction, the material began to refill the nozzle/melt chamber and increased pressure, leading to a gradual recovery of EFW $\left(\Delta \mathrm{W}_{\mathrm{r}}\right)$ to the target width. Another difference found between processing related to retraction and speed was the absence of repetitive oscillation pattern during the recovery after retraction (Figure $8 \mathrm{~b}$ and $\mathrm{c}$ ). This supports the hypothesis that such oscillations were caused by the rapid acceleration of the printhead (for high-speed changes in Figure $7 \mathrm{~b}$ ) and the associated resonance in the machine.

The second set of tests for retraction considered the effect of changing the target EFW and retraction simultaneously (Figure $8 \mathrm{c}$ ). A retraction value of $0.12-0.14 \mathrm{~mm}$ led to the magnitude of the sudden change in filament's width coinciding with its new target width and, therefore, allowed an almost instantaneous transition. For values of retraction below 0.12 $\mathrm{mm}$, the sudden change in EFW was of a lower magnitude than the target change (over- 
extrusion), and vice versa for retraction over $0.14 \mathrm{~mm}$ (under-extrusion). This led to a gradual recovery to the target width after the initial sudden change in both cases.

The obtained data from Figures 7 and 8 were quantitatively characterised to analyse the effect of speed and retraction on EFW in terms of the sudden change $\left(\Delta \mathrm{W}_{\mathrm{s}}\right)$ and recovery $\left(\Delta \mathrm{W}_{\mathrm{r}}\right)$ (Figure 9). Retraction allowed up to $60 \%$ reduction in EFW to be completed over a relatively constant and short distance $(<2 x$ nozzle diameter), as shown in Figure $9 b$, whereas changing speed required a slightly longer distance to achieve similar changes and the required distance increased exponentially for changes in EFW over $40 \%$. The recovery data are plotted alongside the sudden-change data in Figure 9a, separated for recovery after retraction and speed change. Recovery was a much slower process than the sudden change, regardless of whether recovery was instigated by change in speed or retraction. Changing speed resulted in a longer recovery, possibly due to a greater imbalance between the required and actual pressure in the nozzle, whereas retraction recovery happened over a much shorter distance. This information is important from a design point of view, as it can be used as a guidance map to support the choice of which print parameters to vary in order to achieve the desired microscale geometry.

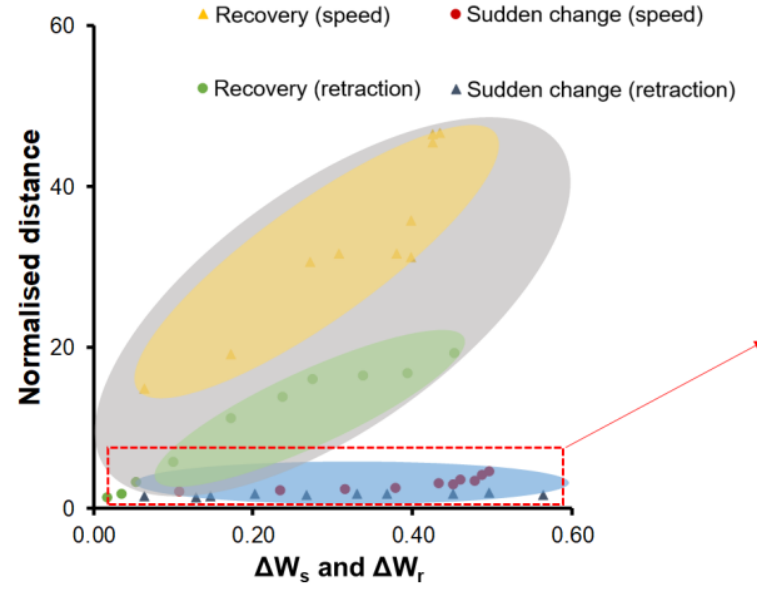

(a)

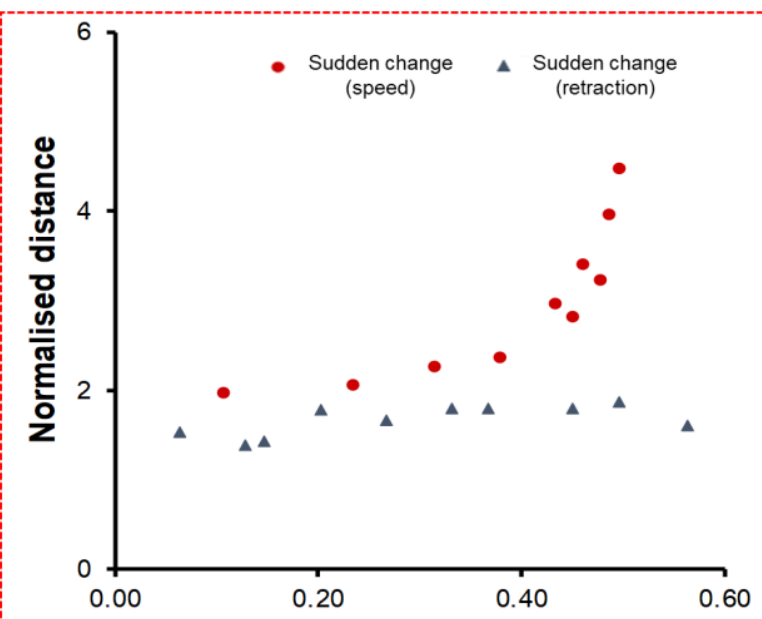

(b)

$\Delta \mathrm{W}_{\mathrm{s}}$

Figure 9 (a) Combined data for normalised distance versus magnitude of normalised EFW change for both sudden reductions and gradual recovery to target EFW. (b) Normalised distance required to achieve different magnitudes of sudden changes to EFW, due to speed-change and retraction.

\subsection{Comparison between direct-drive and Bowden-tube printers}

This section compares the response of the two types of printers in terms of the effect of changes in speed and by retraction/un-retraction on EFW. These comparisons are then repeated with different temperatures and accelerations, to understand the sensitivity and transferability of the findings.

\subsubsection{Print speed and retraction}

A Bowden printer was studied in an equivalent manner to that of the direct-drive printer (Section 3.1). Initially, specimens with a constant target EFW (1.0) along their length were studied. The responses of EFW to the changing speed and retraction/un-retraction are shown in Figure 10a and b, respectively. For simplicity, three levels of low, medium and high 
speeds and retraction values (see Supplementary Data [28]) were selected to compare results for the direct-drive (solid line) and Bowden (dashed line) printers. Additionally, results for sets of specimens with a 50\% lower target EFW in section 2 versus sections 1 and 3 are shown in Figure 10c and d.

As with the direct-drive printer, the Bowden printer demonstrated a reduction in EFW when speed increased, even for a constant target EFW (Figure 10a). This reduction was clearly linked to the magnitude of speed change. However, in stark contrast to direct drive, the Bowden printer did not demonstrate any significant recovery and continued to under-extrude, with measured EFW below the target EFW, over the entire length of section 2 of all specimens, regardless of speed. This can be explained by the large distance between the feeder mechanism and the nozzle (750 $\mathrm{mm}$ compared to $150 \mathrm{~mm}$ for the direct-drive printer), resulting in a lag between changes in achieved and target EFWs. The lag may be caused by the system's slack and slight deformation of the feedstock filament in the Bowden tube, since the compressive forces are transmitted from the feeder along the entire length of the feedstock filament in the tube. A similar conclusion can be obtained by comparing results for the Bowden printer for specimens with constant EFW and with $50 \%$ target EFW in section 2 (Figure 10a and $\mathrm{c}$ ). Whereas the direct-drive printer demonstrated a large difference between these specimen types, the Bowden printer did not, highlighting the unresponsiveness of the latter to the changes in target EFW (as discussed in relation to Figure 6).

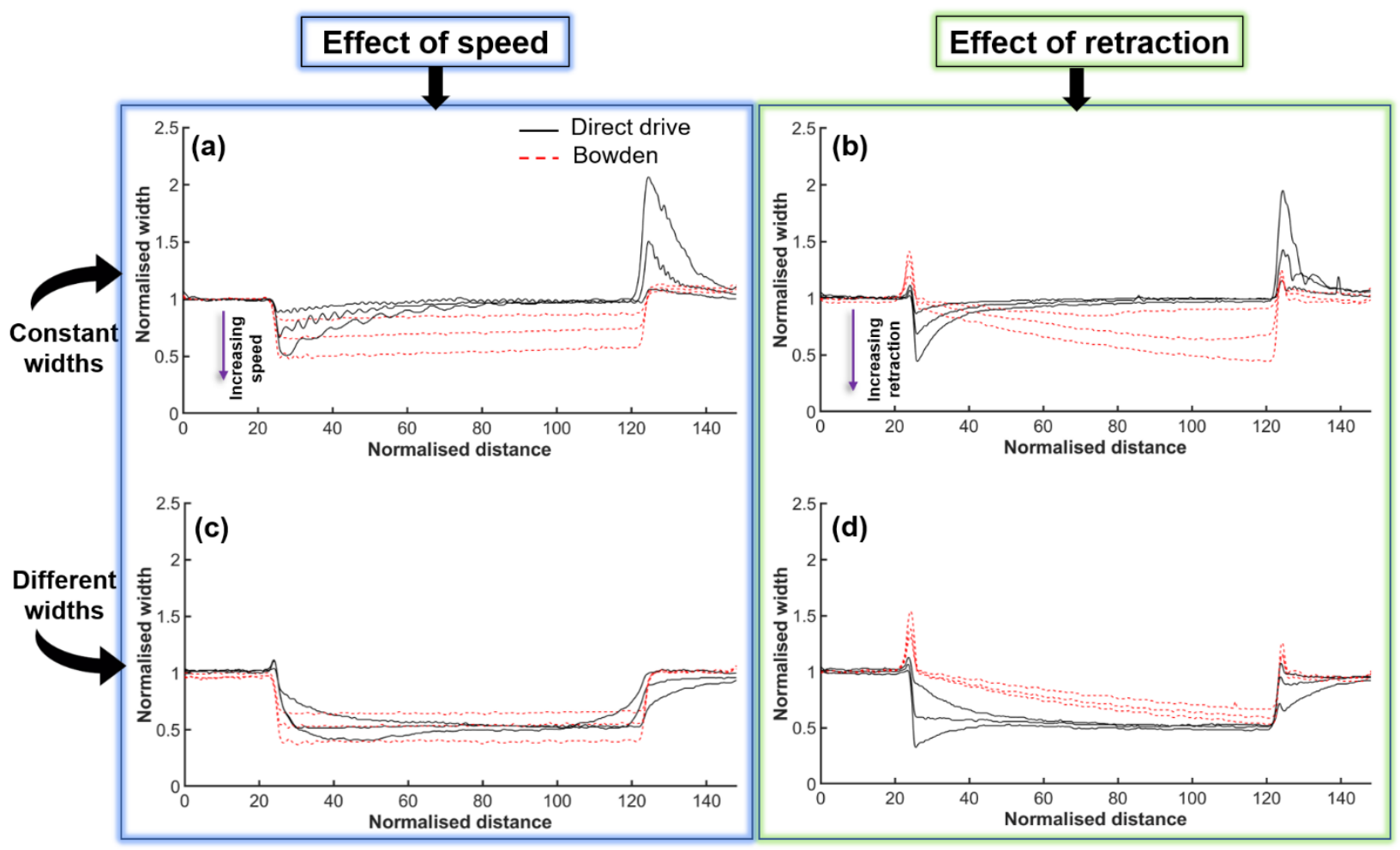

Figure 10 Comparison of the evolution of normalised EFW with normalised distance for direct-drive (solid line) and Bowden tube (dashed line) printers in terms of changing speed ( $a$ and $c$ ) and changing retraction settings ( $b$ and $d$ ). For ( $a$ and $b$ ), target EFW was constant (1.0). For ( $c$ and d), target $E F W$ was halved (0.5) in section 2. 
The Bowden printer was also unresponsive to retraction (Figure 10b and d). Unlike the direct-drive printer, which responded to it with a logical short-term period of under-extrusion, the Bowden printer showed delayed and gradually increasing under-extrusion. The amount of retraction did affect EFW, but even at high values of retraction (beyond those typically used or recommended), EFW did not change responsively. In all cases, the Bowden printer specimens did not recover to the target EFW during the entire length of section 2 (i.e. 100fold distance of nozzle diameter), as can be seen in Figure 10b. Similarly, when target EFW was changed in section 2 (Figure 10d), the Bowden printer did not demonstrate a significant response to retraction.

Overall, it can be concluded that, for Bowden printers, EFW can be responsively influenced by changing print speed, but not by changing the target EFW or retraction, whereas the direct-drive printer was responsive to all three parameters. However, the reduced weight of the printhead for the Bowden printer resulted in a beneficial reduction in EFW oscillation (also called ringing).

\subsubsection{Effect of nozzle temperature}

To test the resiliance of the above results to changes in nozzle temperature, the test specimen with different target EFW in section 2 (defined in Section 2.2) was printed at $195^{\circ} \mathrm{C}, 205^{\circ} \mathrm{C}$ and $215^{\circ} \mathrm{C}$. Effects of print speed changes and retraction were considered. Representative EFW profiles were selected to achieve low, medium and high groupings for speed (Figure 11a and c) and retraction (Figure 11b and d) (see Supplementary Data for print parameters [28]). The plots for changing EFW with retraction are smoother than those for changing speed, indicating the ringing phenomenon discussed in relation to Figure $7 \mathrm{~b}$ that occurred for a wide range of printing conditions. The oscillations were likely caused by vibration of the print-head or printer frame when accelerating to higher speeds. The poor responsiveness of the Bowden printer to changes in extrusion rate was evident at all temperatures, since the target EFW (0.5) was never achieved in section 2. In Figure 11c, it appears that the material's volumetric flowrate from the Bowden printer's nozzle did not change throughout the entire specimen (even though the set rate varied in G-Code). This mean that the speed is a highly effective parameter to control EFW for the Bowden printer, as demonstrated in case studies in Section 3.4. Overall, the results show that trends found in this study were also observed for a range of extrusion temperatures, giving confidence in the applicability and resilience of the CONVEX design approach for both direct-drive and Bowden printers.

\subsubsection{Effect of acceleration}

The previous sections demonstrated the important effect that speed has on extrudedfilament geometry. Since acceleration directly affects speed, a range of accelerations were studied for 1.0-3.0-1.0 specimens. For both the direct-drive and Bowden printers, it was possible to achieve a gradual reduction in EFW at lower accelerations and a sudden reduction at higher accelerations (Figure 12). The Bowden printer (Figure 12b) demonstrated a controllable gradual change in EFW at both the beginning (reducing EFW) and the end (increasing EFW) of section 2. The direct-drive printer (Figure 12a) demonstrated a more complicated trend, potentially resulting from attempts by the system firmware to compensate for over-extrusion. The effect of jerk was also investigated but was found to have no significant effect on EFW profiles, so the results are not shown here. The results for acceleration confirm that it is an important parameter that can be used to control the transition between different EFWs. This is demonstrated in a case study in Section 3.4. 


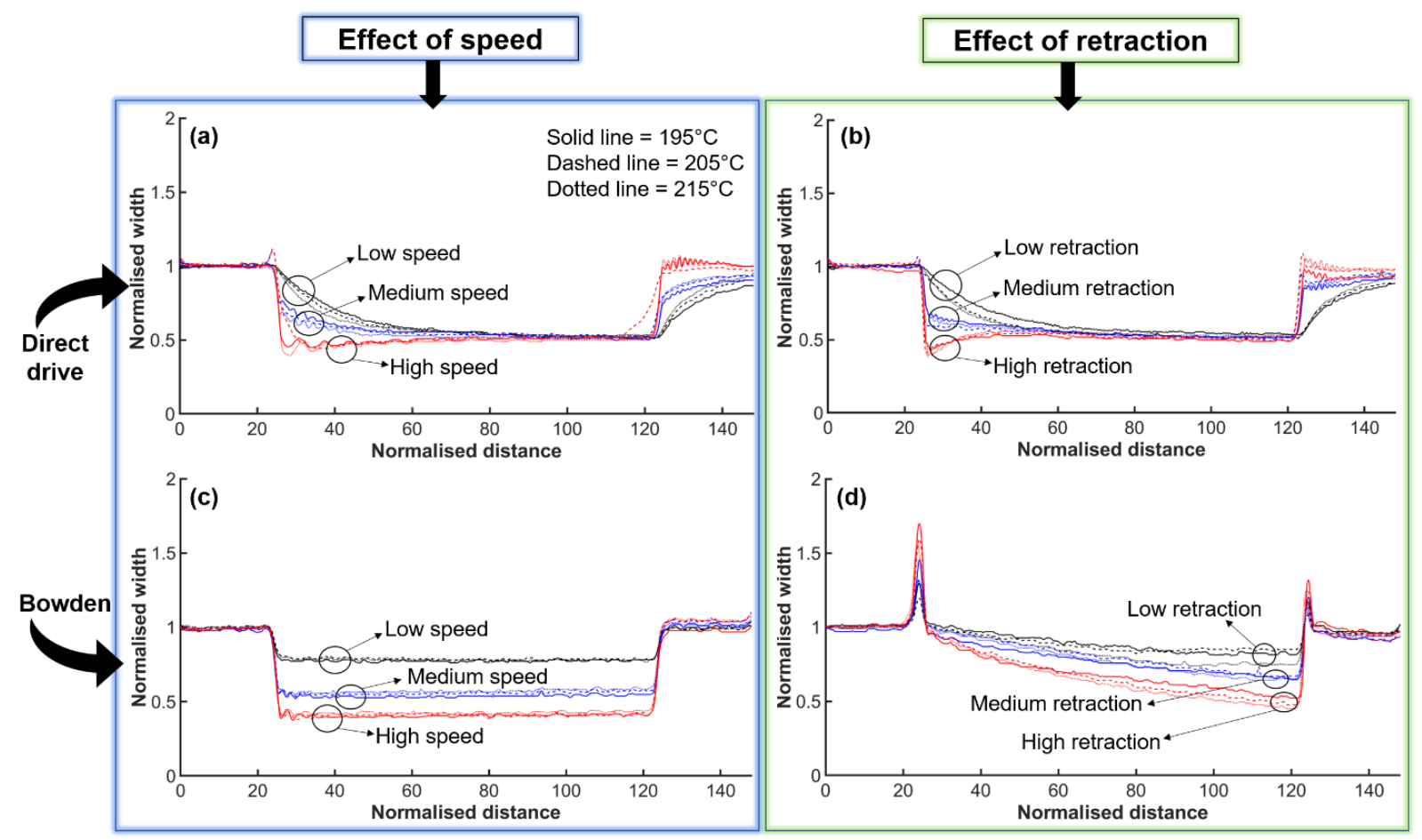

Figure 11 Effect of different temperatures on filament geometry for direct-drive and Bowden tube printers. ( $a$ and c) Effect of three levels of speed. ( $b$ and d) Effect of three levels of retraction. Target EFW was 1.0 in sections 1 and 3 and 0.5 in section 2.
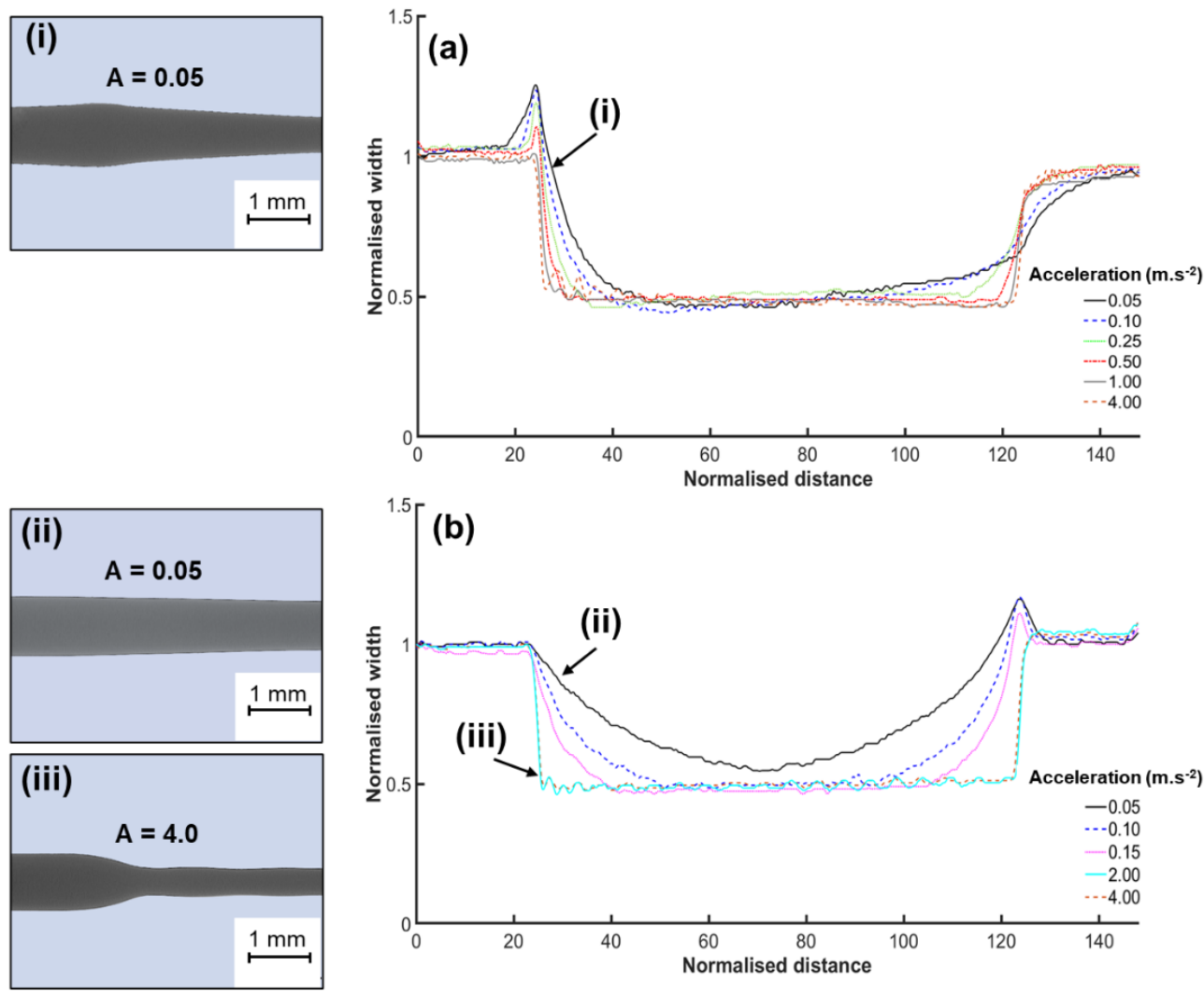

Figure 12 Effect of changing acceleration on normalised EFW for both direct-drive (a) and Bowden (b) printers. Inset optical images with gradual (i and ii; acceleration $0.05 \mathrm{~m} . \mathrm{s}^{-2}$ ) and sudden (iii; acceleration $4.0 \mathrm{m.s}^{-2}$ ) reduction in width. Target EFW was 1.0 in sections 1 \& 3 and 0.5 in section 2 . 


\subsection{Alternative materials and layer height}

The transferability of results obtained in Sections 3.1 and 3.2 was investigated in terms of material choice: PLA, ABS and nylon. By changing speed in section 2 of the specimen, it was possible to achieve similar reductions in EFW for all three materials (Figure 13a). Furthermore, this was possible for a range of EFW reductions for all materials (from $30 \%$ to $70 \%$ reduction) as can be seen by the groupings into low, medium and high speeds in section 2 of the specimens (print parameters given in Supplementary data [28]). Based on these results, it is expected that the findings of this study are applicable to a wide range of materials. In addition to investigate the applicability of results to other layer heights, the effect of print speed at a lower layer height $(0.1 \mathrm{~mm})$ was also investigated (Figure 13b). The obtained result was compared against the reference layer height of $0.2 \mathrm{~mm}$ and shows the feasibility of controlling EFW with different layer heights. The level of speed is still an effective parameter for controlling target EFW when layer height is reduced by $50 \%$ (see Supplementary Data for print speed values [28]).

A full comparison of the gradient of the sudden change upon changing different parameters for both the direct-drive and Bowden printers is presented in Figure 14. For major parameters, including changing speed and retraction, the direct-drive printer (Figure 14a and c) appeared to be more responsive because a wide range of magnitudes of EFW changes were achieved in a normalised distance of approximately 1 to 3 (equivalent to 1-3x nozzle diameter) for most parameters, whereas the Bowden printer achieved similar EFW changes in a normalised distance of approximately 2 to 5 . Also, retraction did not achieve controllable changes to EFW for the Bowden printer (even for a normalised distance of 100), so does not feature in Figure 14b and $\mathrm{d}$. For both printers, acceleration clearly enabled the normalised distance for changes to EFW to be increased by up to ten-fold.
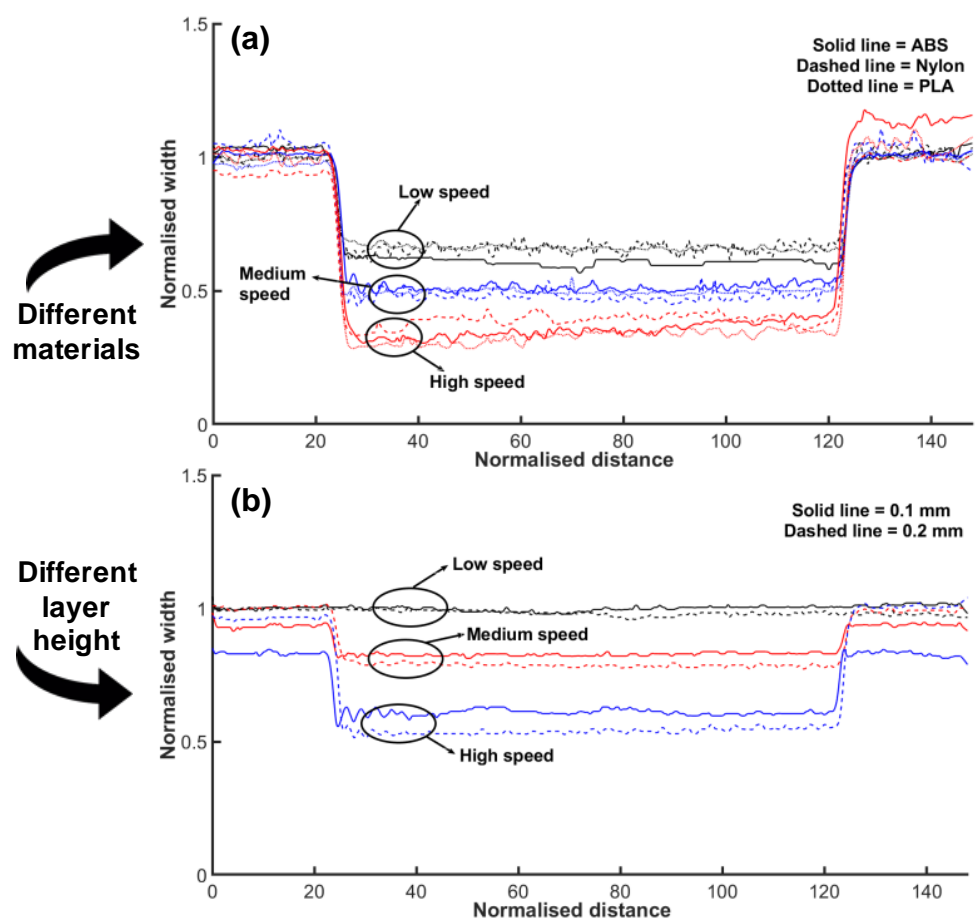

Figure 13 (a) Control of normalised EFW for three different materials ( $P L A, A B S$ and nylon) by adjusting speed in section 2 of specimens. (b) Comparison between specimens with 0.1- and 0.2-mm layer heights and three levels of print speed. Target EFW was 1.0 in sections 1 and 3 and 0.5 in section 2. 

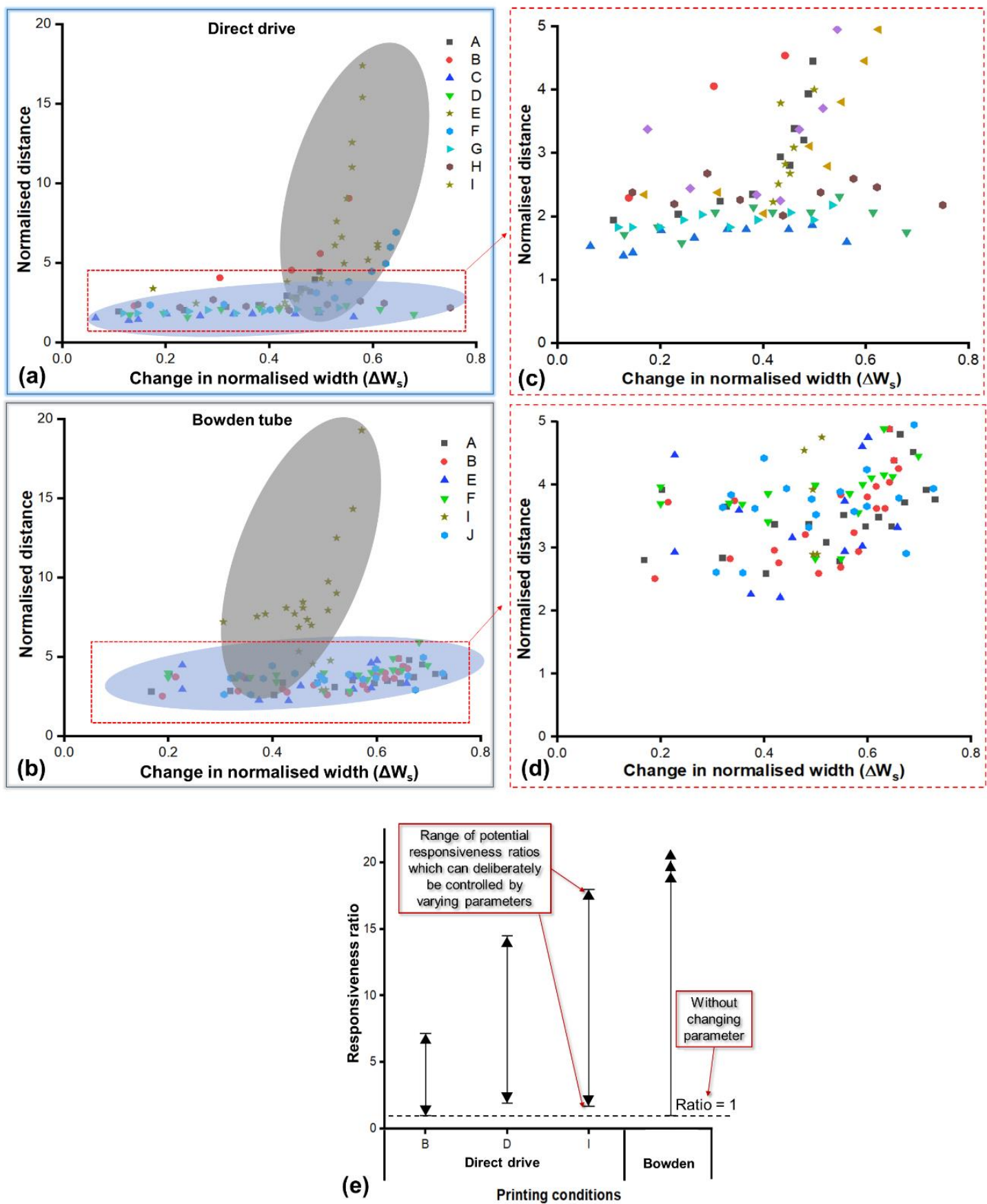

Figure 14 Comparison of the sudden change in normalised EFW for different conditions printed by the direct-drive (a) and Bowden (b) printers. (c and d) zoomed-in insets for direct-drive and Bowden tube. (e) Range of possible responsiveness ratios for different printing conditions considered in this study. A: change speed only; B: change speed + target EFW; C: retraction only; $D$ : retraction + target EFW; E/F: speed + target EFW at $195 / 215^{\circ} \mathrm{C} ; \mathrm{G} / \mathrm{H}$ : retraction + target EFW at $195 / 215^{\circ} \mathrm{C}$; I: speed + target EFW + acceleration; J: different speed + target EFW + different materials.

Furthermore, for specimens set to have a $50 \%$ reduction of EFW in section 2, the normalised distance necessary to achieve this reduction was calculated for cases when (i) the extrusion rate was changed only, and (ii) the extrusion rate was changed in combination with the speed change or retraction. The responsiveness ratio, which is the ratio of normalised distance required for case (i) divided by that for case (ii), was calculated to assess the responsiveness of the printers for different printing parameters: a ratio of 10 indicates a ten- 
fold reduction in the distance required to achieve the new target EFW. The wide range in achievable ratios (from 1 to $>20$ ), as shown in Figure 14e, demonstrates how broadly the geometry of the filament can be controlled by the methods considered in this study. For the Bowden printer, the target width was never achieved at a normalised distance of 100; thus, responsiveness ratio could not be measured but it was much greater than 20 (potentially higher than 100).

\subsection{Industrial applicability and case studies}

Immediate industrial relevance: The method developed in this study to characterise the dynamic response of extrusion process to changes in printer parameters has direct industrial relevance for hardware development and validation. By repeating some aspects of this study with different extruder/feeder hardware, it is possible to quantitatively compare performance in terms of responsiveness, since many extruder/feeder mechanisms strive to optimise responsiveness for aspects such as stopping/starting extrusion with retraction. Similarly, the CONVEX approach used in this study can be employed for development of the new material formulations. The understanding can also be implemented in 3D printing software to better optimise the print path and print parameters based on the printer hardware (e.g. direct drive versus Bowden).

Other manufacturing processes: Although this study considers the MEAM process, it may be possible to translate the CONVEX design concept to other manufacturing processes. For laser etching, cutting or surface treatment, the laser's scanning speed could be controlled, in a similar way to the nozzle's travel speed in this study, to change the energy density and achieve varying surface treatment features or kerf widths [33]. A key consideration for translation to other AM processes is whether layer thickness unavoidably varies when width changes; MEAM overcomes this issue because the flat bottom of the nozzle constrains the layer thickness even when EFW changes. For laser-based processes, the penetration depth would vary, which would affect the mechanical properties. Still, there are research opportunities to investigate the potential to use CONVEX in other AM processes to design geometries below the scale of nominal smallest features (e.g. laser track width / material jetting path).

Design potential: The findings presented in the above results sections highlight the potential to design structures for MEAM using the CONVEX design approach to continuously and controllably vary the geometry of individual filaments. In terms of design freedom, the results in Figure $7 d$ show the potential to design continuous extrusions with varying geometries across a range at least from $75 \%$ to $250 \%$ of the nozzle diameter. Even the most advanced capabilities within current "slicer" software packages and current research studies (e.g. setting different values for EFW) do not consider design size scales similar to that used in this study (e.g. designed and continuous variation of EFW). The newly demonstrated ability to control geometry of a single extruded filament while it is being printed (through combinations of speed, acceleration, retraction and the set extrusion rate) opens up new possibilities for fields ranging from next generation slicers to completely new structures (e.g. new tissue engineering scaffolds, drug delivery constructs, filters, mechanically graded infill lattices and microfluidic mixers). Step-change capabilities are possible with CONVEX for even the most recent state-of-the-art printing developments - for example free-standing pillar arrays [18] could be given explicitly designed and continuously varying diameters. 
Furthermore, the results demonstrating applicability of the findings to different extrusion temperatures, materials (PLA, ABS, nylon) and layer heights, indicate the robustness and transferability of the method.

Application and case studies: Due to the complexity of the design of intricate structures, the CONVEX design approach is most applicable to research fields and high-value applications, where MEAM is being used to develop new metamaterials and microstructural geometries or to manufacture final products/specimens. However, integration of the new understanding into slicers may allow a more widespread use for larger-scale manufacturing. The first case study below demonstrates the use of the CONVEX design approach in slicing algorithms to eliminate pore defects. The second case study shows how CONVEX may enable the design of structures at sub-filament size scales (i.e. with features smaller than the width of extruded filaments) and the third case study demonstrates the potential for entirely new structurematerial designs. All case studies used the Bowden printer to highlight the capabilities of the CONVEX approach even for less responsive printer hardware (compared to direct-drive printers).

\subsubsection{Case study 1: "Streamline Slicing" application of CONVEX}

In this case study, a rectangular tab with a tapered central section (shown schematically in Figure 15a) was printed using two approaches: (i) conventional slicing software; (ii) the CONVEX design approach with a streamlined print path (produced using in-house software for GCODE generation).

For the version produced by slicing software, the printing direction in the slicer was set to be in the $Y$ direction, (dashed arrow in Figure 15a), which is the logical direction to achieve a neat manufactured part. For the CONVEX version, the understanding developed in this study was used to continuously vary EFW to match the overall design geometry. To achieve this gradual narrowing of the extruded filaments, the magnitudes of speed and acceleration were deliberately controlled (as highlighted in Figure 15b), based on results related to acceleration and EFW in Figure 12b.

For the part produced with conventional slicing software, the perimeter of the design (i.e. solid shell) was printed first, and then the remaining internal space was filled by an array of filaments. Since the print speed and EFW were set by the slicing software at constant values for each extruded filament, once the nozzle reached the transition point (i.e. reduction in width meant fewer filaments were required to fill the space), it stopped and moved to the next line. This resulted in multiple voids (Figure 15b, $d$ and f) with sizes in the range of 400 $\mu \mathrm{m}$, similar to those found in the literature which cause stress concentration and premature failure $[25,26]$. In contrast, since the CONVEX design utilised a constant number of extruded filaments following the streamlined paths along the entire length of the part, there were no voids or defects (Figure 15c and e).

This case study demonstrated a specific implementation of the CONVEX design approach, based around the idea of "Streamline Slicing", where the understanding developed in this study allowed EFW to be varied along a streamlined print path. It is particularly relevant for parts with simple but non-uniform geometry that have stringent requirements for mechanical integrity, effective sealing (no pore defects) for fluid application or pneumatics, or aesthetics. Practically, this could be implemented in slicing software by allowing the user to specify the streamline orientation or by selecting two faces of the CAD model to prescribe the start and end of streamlines. 

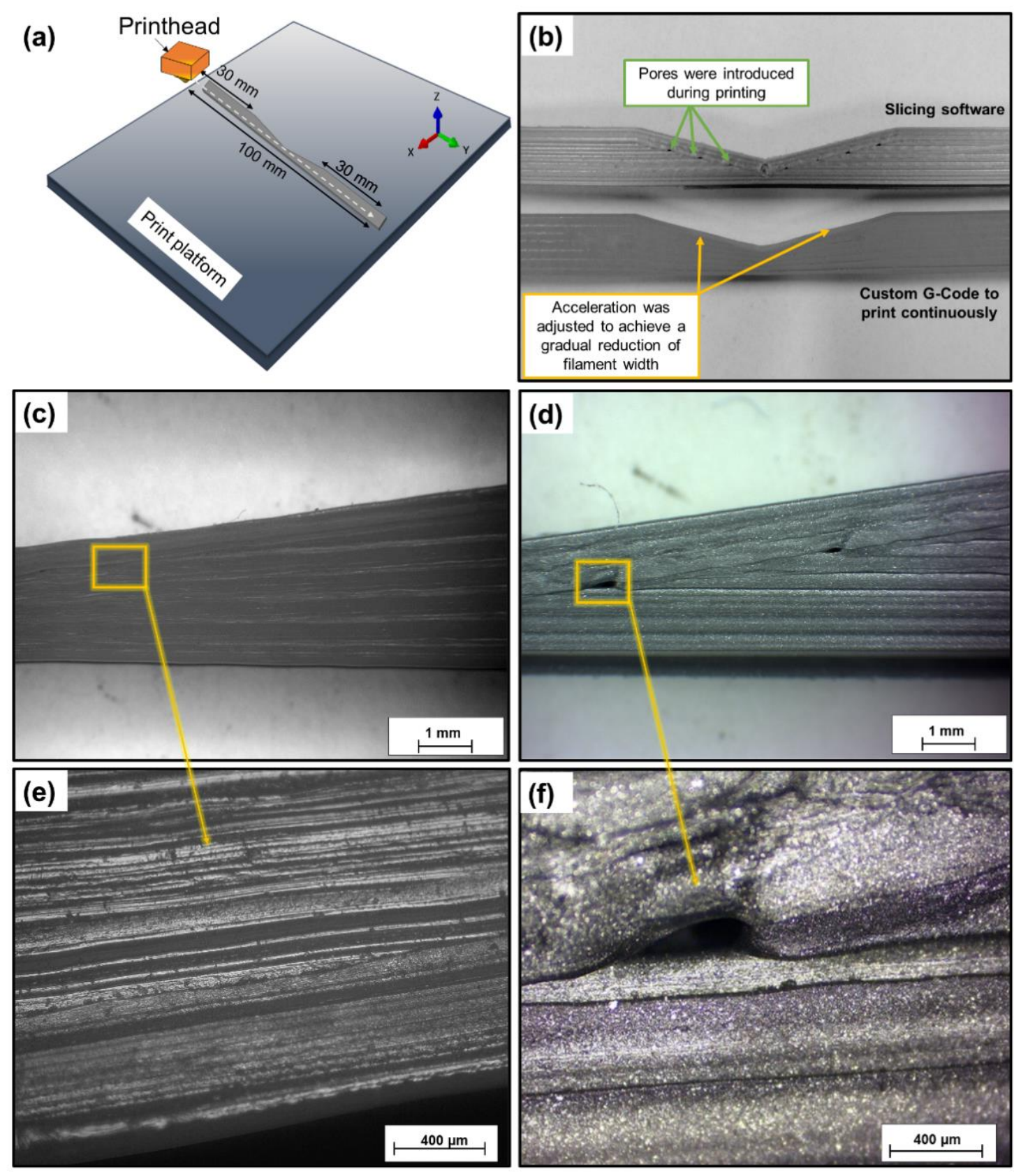

Figure 15 (a) Schematic of print-platform orientation of demonstration part. (b) Parts manufactured with conventional slicing software and CONVEX design approach. ( $d$ and f) Multiple pore defects in specimens printed with conventional slicing software. (c and e) No defects for CONVEX approach.

\subsubsection{Case study 2: microscale geometric design application of CONVEX}

For the second case study, a micro-tensile testing specimen was used to demonstrate the potential to design structures with design features smaller than the extruded filament. Currently, there is no standard for MEAM structure for tensile testing, and many research groups use ASTM D638 standard to print dogbone specimens [16,25]. However, a critical issue with this design is the presence of void or seams between the extruded filaments, similar to those in the previous case study (Figure 15). To overcome these issues, a filament-scale dogbone specimen (Figure 16a) was printed, using the understanding from the results in Section 3.1 (Figure 7d) to print the wide (grips) and narrow (gauge) sections in a single pass (Figure 16a and b). The print speed for the gauge section of specimen was 
increased from 1.0 to $3.0 \mathrm{~m} \cdot \mathrm{min}^{-1}$ to quickly adjust the filament width from the grips-section width to the gauge width.

For comparison, a CAD model of the design was used to print the same geometry with the conventional slicing software. The part produced by conventional slicing software (Figure 16d) contained void defects between the filaments similar to those described in the literature $[16,25]$, whereas the part manufactured using the CONVEX design approach demonstrated neat and continuous extrusion (Figure 16c). This micro-tensile testing specimen, based on the CONVEX design approach, enabled several research studies by the authors to develop new fundamental understanding of mechanical performance in MEAM. It was possible to print the dogbone geometry for 225 layers $(45 \mathrm{~mm}$ high) without distortions or warping, and with tensile strength equivalent to that of the bulk polymer, highlighting that the CONVEX approach does not introduce critical residual stresses [24,27]. These studies would not have been possible with conventional testing specimens and print-path approaches, and the method is currently being translated to other fields.

There is a huge range of structures currently designed based on an unnecessarily assumed limitation that filaments need a constant cross-section (e.g. tissue engineering scaffolds, filters, fluid mixers, drug delivery constructs, infill patterns and mechanical lattices). By reconsidering the geometric design of such structures based on the CONVEX design approach, a step change in MEAM capability and understanding is possible.

(a)

(b)

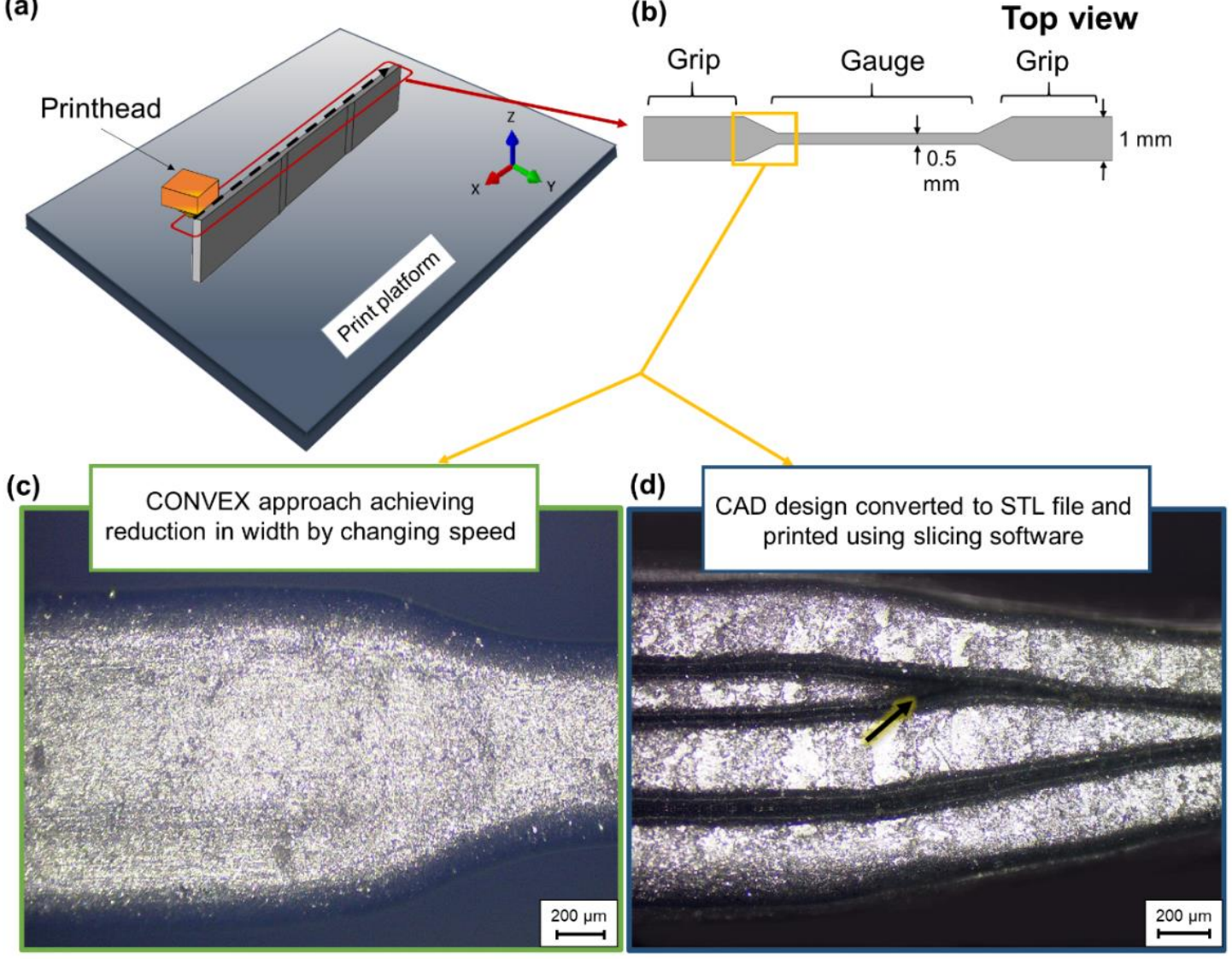

Figure 16 (a) Schematic of microscale dogbone tensile-testing specimen on the print platform. (b) Top-view schematic of the specimen. Micrographs of parts produced with custom G-Code based on CONVEX design approach (c) or with slicing software (d; with pore defects shown by arrow and interfilament seams). 


\subsubsection{Case study 3: microscale geometrically graded mesh materials}

A final case study demonstrates MEAM mesh materials with graded pore sizes that are inconceivable and impossible to produce without CONVEX (Figure 17). Grading is normally only possible through varying the geometry and position of an entire filament; CONVEX allows entire new spatial freedom for design of mesh materials. In the case study, for different specimens, print speed was set constant at $1.0 \mathrm{~m}^{\mathrm{min}}{ }^{-1}$ (Figure 17b) or varied from 0.5 to $2.0 \mathrm{~m}^{\mathrm{min}} \mathrm{m}^{-1}$ according to linear and sinusoidal functions (Figure 17c-f). EFW was set to be constant $(0.5 \mathrm{~mm})$ for the traditional mesh and varied up to 2-fold for the graded mesh materials. A video of the printing process for these specimens is included as supplementary data [28]. Since the CONVEX design approach allows geometric grading without changing the print path, it is possible to instantly vary grading for each layer whilst still ensuring effective contact with filaments on lower layers. Conversely, for constrained print paths (e.g. conforming to anatomical geometry) CONVEX offers new opportunities to control pore geometry. Since this case study used a 50\% larger nozzle than the rest of the study, it also highlights the potential of CONVEX for alternative size-scales (e.g. small needles for biomedical applications or large nozzles for construction and other large-scale MEAM).

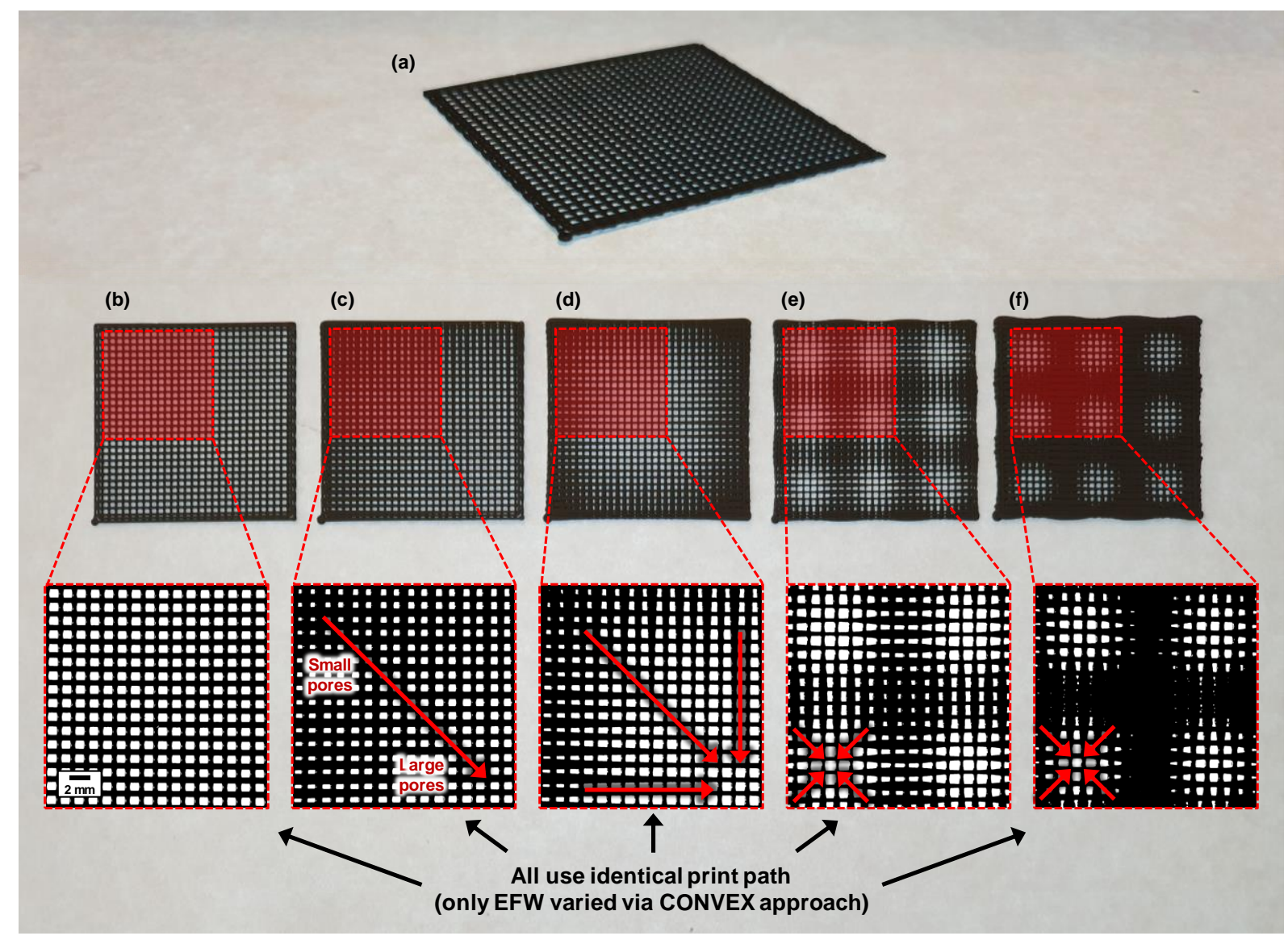

Figure 17 (a) Photo of mesh material specimen. Five designs were printed with and without the CONVEX design approach with identical print path: (b) traditional mesh design with constant EFW; (c) linearly graded continuous variation of EFW; (d) sinusoidally graded EFW; (e) one-third-period sinusoidally graded EFW; (f) similar to (e) with 67\% wider EFW. (Arrows indicate grading direction for pore-size enlargement. Videos of the variable-speed printing process are included in supplementary data [28]. The scale bar is the same for all five inset images.) 


\section{Conclusions}

The effect of print speed, retraction and acceleration on the geometry of single extruded filaments was studied for two main classes of MEAM 3D printers (direct-drive and Bowden). The results demonstrated that by changing these parameters in a designed way, the geometry of the extruded filament could be controlled and varied along its length. The obtained results indicated that direct-drive printers were more responsive. In particular, retraction had a little effect on the Bowden printer. Furthermore, for both printer types, it was possible to achieve similar changes to extruded-filament geometry for a range of materials (PLA, ABS and nylon), at temperatures $10^{\circ} \mathrm{C}$ above and below the recommended printing temperature, and for different layer heights and nozzle sizes. Acceleration was used to directly affect the rate of transitions between wider and narrower extruded-filament geometries. Three case studies demonstrated the use of the newly developed understanding in the CONVEX design approach, in which the width of extruded filaments is explicitly designed to achieve structures that are not possible using a conventional slicer. The new approach opens up a new size scale for design (e.g. tens of microns design resolution for a $0.4 \mathrm{~mm}$ nozzle) and the opportunity for a step change in novel structural geometries as well as new potential for future 3D printing slicer software.

\section{Acknowledgements}

This research did not receive any specific grant from funding agencies in the public, commercial, or not-for-profit sectors.

\section{References}

[1] J.W. Stansbury, M.J. Idacavage, 3D printing with polymers: Challenges among expanding options and opportunities, Dent. Mater. 32 (2016) 54-64. https://doi.org/https://doi.org/10.1016/j.dental.2015.09.018.

[2] L. Safai, J.S. Cuellar, G. Smit, A.A. Zadpoor, A review of the fatigue behavior of 3D printed polymers, Addit. Manuf. 28 (2019) 87-97. https://doi.org/10.1016/j.addma.2019.03.023.

[3] S.C. Ligon, R. Liska, J. Stampfl, M. Gurr, R. Mülhaupt, Polymers for 3D printing and customized additive manufacturing, Chem. Rev. 117 (2017) 10212-10290. https://doi.org/10.1021/acs.chemrev.7b00074.

[4] L.G. Bracaglia, B.T. Smith, E. Watson, N. Arumugasaamy, A.G. Mikos, J.P. Fisher, 3D printing for the design and fabrication of polymer-based gradient scaffolds, Acta Biomater. 56 (2017) 3-13. https://doi.org/10.1016/j.actbio.2017.03.030.

[5] C. Culmone, G. Smit, P. Breedveld, Additive manufacturing of medical instruments: A state-of-the-art review, Addit. Manuf. 27 (2019) 461-473. https://doi.org/10.1016/j.addma.2019.03.015.

[6] U.K. uz Zaman, E. Boesch, A. Siadat, M. Rivette, A.A. Baqai, Impact of fused deposition modeling (FDM) process parameters on strength of built parts using Taguchi's design of experiments, Int. J. Adv. Manuf. Technol. 101 (2019) 1215-1226. https://doi.org/10.1007/s00170-018-3014-6.

[7] W. Zhu, K.R. Tringale, S.A. Woller, S. You, S. Johnson, H. Shen, J. Schimelman, M. Whitney, J. Steinauer, W. Xu, T.L. Yaksh, Q.T. Nguyen, S. Chen, Rapid continuous 3D printing of customizable peripheral nerve guidance conduits, Mater. Today. 21 (2018) 951-959. https://doi.org/10.1016/j.mattod.2018.04.001.

[8] S. Petersmann, M. Spoerk, W. Van De Steene, M. Üçal, J. Wiener, G. Pinter, F. Arbeiter, Mechanical properties of polymeric implant materials produced by extrusion-based additive manufacturing, J. Mech. Behav. Biomed. Mater. 104 (2020) 103611. https://doi.org/10.1016/j.jmbbm.2019.103611.

[9] P. Geng, J. Zhao, W. Wu, W. Ye, Y. Wang, S. Wang, S. Zhang, Effects of extrusion speed and printing speed on the 3D printing stability of extruded PEEK filament, J. Manuf. Process. 37 (2019) 266-273. https://doi.org/10.1016/j.jmapro.2018.11.023.

[10] C. Pascual-gonzález, M. Iragi, A. Fernández, J.P. Fernández-blázquez, L. Aretxabaleta, An approach to analyse the factors behind the micromechanical response of 3D-printed composites, Compos. Part B. 186 (2020) 107820. https://doi.org/10.1016/j.compositesb.2020.107820.

[11] Y. Zhang, C. Purssell, K. Mao, S. Leigh, A physical investigation of wear and thermal characteristics of 3D printed nylon spur gears, Tribol. Int. 141 (2020) 105953. https://doi.org/10.1016/j.triboint.2019.105953.

[12] A. Gleadall, W. Poon, J. Allum, A. Ekinci, X. Han, V. V. Silberschmidt, Interfacial fracture of 3D-printed bioresorbable polymers, Procedia Struct. Integr. 13 (2018) 625-630. 
https://doi.org/https://doi.org/10.1016/j.prostr.2018.12.103.

[13] A. Gleadall, D. Visscher, J. Yang, D. Thomas, J. Segal, Review of additive manufactured tissue engineering scaffolds: relationship between geometry and performance, Burn. Trauma. 6 (2018) 1-16. https://doi.org/10.1186/s41038-018-0121-4.

[14] V.E. Kuznetsov, A.G. Tavitov, O.D. Urzhumtsev, M. V. Mikhalin, A.I. Moiseev, Hardware factors influencing strength of parts obtained by fused filament fabrication, Polymers (Basel). 11 (2019) 1870. https://doi.org/10.3390/polym11111870.

[15] A. Sood, R. Ohdar, S. Mahapatra, Parametric appraisal of mechanical property of fused deposition modelling processed parts, Mater. Des. 31 (2010) 287-295. https://doi.org/https://doi.org/10.1016/j.matdes.2009.06.016.

[16] B. Rankouhi, S. Javadpour, F. Delfanian, T. Letcher, Failure analysis and mechanical characterization of 3D printed ABS with respect to layer thickness and orientation, J. Fail. Anal. Prev. 16 (2016) 467-481. https://doi.org/10.1007/s11668-016-0113-2.

[17] J.C. Riddick, M.A. Haile, R. Von Wahlde, D.P. Cole, O. Bamiduro, T.E. Johnson, Fractographic analysis of tensile failure of acrylonitrile-butadiene-styrene fabricated by fused deposition modeling, Addit. Manuf. 11 (2016) 49-59. https://doi.org/10.1016/j.addma.2016.03.007.

[18] B. Nan, F.J. Galindo-Rosales, J.M.F. Ferreira, 3D printing vertically: Direct ink writing free-standing pillar arrays, Mater. Today. 35 (2020) 16-24. https://doi.org/10.1016/j.mattod.2020.01.003.

[19] F. Tamburrino, S. Graziosi, M. Bordegoni, The influence of slicing parameters on the multi-material adhesion mechanisms of FDM printed parts: an exploratory study, Virtual Phys. Prototyp. 14 (2019) 316332. https://doi.org/10.1080/17452759.2019.1607758.

[20] E. Nyberg, A. O'Sullivan, W. Grayson, ScafSlicr: A MATLAB-based slicing algorithm to enable 3D-printing of tissue engineering scaffolds with heterogeneous porous microarchitecture, PLoS One. 14 (2019) e0225007. https://doi.org/10.1371/journal.pone.0225007.

[21] J.M. Barrios, P.E. Romero, Decision tree methods for predicting surface roughness in fused deposition modeling parts, Materials (Basel). 12 (2019) 2574. https://doi.org/10.3390/ma12162574.

[22] J. Wang, H. Xie, Z. Weng, T. Senthil, L. Wu, A novel approach to improve mechanical properties of parts fabricated by fused deposition modeling, Mater. Des. 105 (2016) 152-159. https://doi.org/10.1016/..matdes.2016.05.078.

[23] H.I. Medellin-Castillo, J. Zaragoza-Siqueiros, Design and manufacturing strategies for fused deposition modelling in additive manufacturing: A review, Chinese J. Mech. Eng. (English Ed. 32 (2019) 1-16. https://doi.org/10.1186/s10033-019-0368-0.

[24] J. Allum, A. Moetazedian, A. Gleadall, V. V Silberschmidt, Interlayer bonding has bulk-material strength in extrusion additive manufacturing: new understanding of anisotropy, Addit. Manuf. 34 (2020) 101297. https://doi.org/10.1016/j.addma.2020.101297.

[25] J. Lluch-Cerezo, R. Benavente, M.D. Meseguer, S.C. Gutiérrez, Study of samples geometry to analyze mechanical properties in Fused Deposition Modeling process (FDM), Procedia Manuf. 41 (2019) 890-897. https://doi.org/10.1016/j.promfg.2019.10.012.

[26] J.R.C. Dizon, A.H. Espera, Q. Chen, R.C. Advincula, Mechanical characterization of 3D-printed polymers, Addit. Manuf. 20 (2018) 44-67. https://doi.org/10.1016/j.addma.2017.12.002.

[27] A. Moetazedian, A. Gleadall, X. Han, V.V. Silberschmidt, Effect of environment on mechanical properties of 3D printed polylactide for biomedical applications, J. Mech. Behav. Biomed. Mater. 102 (2020) 103510. https://doi.org/https://doi.org/10.1016/j.jmbbm.2019.103510.

[28] A. Gleadall, A. Moetazedian, A. Budisuharto, V. V. Silberschmidt, Data for article - CONVEX (CONtinuously Varied EXtrusion): a new scale of design for additive manufacturing, (2020). https://figshare.com/s/1fbdd3609e9e951214d1.

[29] P. Gharge, Velocity painting: paint your 3D prints with style, All3DP. (2020). https://all3dp.com/2/velocitypainting-how-to-paint-your-3d-prints-with-style/ (accessed February 3, 2020).

[30] G. Hsiang Loh, E. Pei, J. Gonzalez-Gutierrez, M. Monzón, An Overview of Material Extrusion Troubleshooting, Appl. Sci. 10 (2020) 4776. https://doi.org/10.3390/app10144776.

[31] A. Derossi, M. Paolillo, R. Caporizzi, C. Severini, Extending the 3D food printing tests at high speed. Material deposition and effect of non-printing movements on the final quality of printed structures, J. Food Eng. 275 (2020) 109865. https://doi.org/10.1016/j.jfoodeng.2019.109865.

[32] S. Abdollahi, A. Davis, J.H. Miller, A.W. Feinberg, Expert-guided optimization for 3D printing of soft and liquid materials, PLoS One. 13 (2018) 1-19. https://doi.org/10.1371/journal.pone.0194890.

[33] B. Yilbas, Laser cutting quality assessment and thermal efficiency analysis, J. Mater. Process. Technol. 155-156 (2004) 2106-2115. https://doi.org/https://doi.org/10.1016/j.jmatprotec.2004.04.194. 